\title{
The stability and the nonlinear evolution of quasi-geostrophic hetons
}

\author{
Jean N. Reinaud ${ }^{1,{ }^{*}}$ and Xavier Carton $^{2}$ \\ ${ }^{1}$ Mathematical Institute, University of St Andrews, North Haugh, St Andrews, KY16 9SS, UK \\ ${ }^{2}$ Laboratoire de Physique des Océans, UFR Sciences, UBO/UEB, 6, Avenue le Gorgeu, 29200 Brest, France \\ *: Corresponding author : Jean N. Reinaud, email address : jean@mcs.st-and.ac.uk
}

\begin{abstract}
:
We analyse the linear stability and nonlinear evolutions of circular hetons under the quasi-geostrophic approximation. We compare results obtained with a three-layer model and with a model based on a continuous density stratification. Though the models also differ by the vertical boundary conditions, they show a remarkable similarity in the stability properties of the hetons (threshold values of vortex radius for baroclinic instability, dominant modes, growth rates, etc.), and in their nonlinear evolutions (spatial reorganization of potential vorticity by nonlinear processes, end-states of the simulations). The hetons prone to baroclinic instability often break into two hetons drifting in opposite directions, and in more hetons, for wider initial structures. In both models, instability is quite sensitive to the vertical gap between the opposite-signed vortices: as it increases, the instability decreases and shifts to lower azimuthal modes. Finally, though modes $I \geq 2$ (i.e. elliptical and shorter wave deformations) prevail in most of the parameter space, the mode $I=1$ perturbation (a vertical tilt of the vortex column) exists for hetons with small vertical gaps. Such perturbations are concentrated vertically near the gap, and can only be evidenced in the continuously stratified model.
\end{abstract}

\section{Introduction}

Vortices, which are long-lived coherent structures, abound in the oceans where they play an important role in momentum, heat and tracer fluxes. The vortices which were most intensively studied originally were the Gulf-Stream rings (see a review of these studies in Robinson (1983)). Indeed, the mixed barotropic-baroclinic instability of this intense eastward jet leads to the formation of large vortices (called rings) via the occlusion of its meanders. Though the warm-core and cold-core Gulf-Stream rings were observed most often, deep anticyclones of Sargasso Sea water were found to form in conjunction with cold-core rings (Bane, O'Keefe \& Watts (1989)) and to propagate away from the GulfStream as coherent, baroclinic vortex pairs. The characteristic horizontal size of these dipoles is between 150 and $250 \mathrm{~km}$. Baroclinic vortex dipoles were also observed recently in the Eastern North Atlantic Ocean, forming from the Mediterranean Water undercurrents in the Gulf of Cadiz (Carton et al., 2002, Serra, Ambar \& K"ase, 2005). In that occurrence also, the cyclones lie above the anticyclones and the coupled vortices can propagate southward away from their region of generation. The horizontal size of these dipoles is between 80 and $120 \mathrm{~km}$. In both cases (Gulf-Stream region and Gulf of Cadiz), the first internal radius of deformation is about $30-35 \mathrm{~km}$. This radius of deformation characterises the equal influence of stable stratification and of Earth rotation on these mesoscale motions. The simplest dynamical model which takes into account the dominant 
effects of rotation and stratification, is the quasi-geostrophic model. Quasi-geostrophic vortices can interact with one another, and vortex interaction has been extensively studied in the literature.

Vortex interactions were first studied when vortices were located at the same depth. An important class of such vortex interactions, which received much attention in the past, is vortex merger. Vortex merger is the process by which two co-rotating vortices, at the same depth or which overlap vertically, collapse towards their mutual centre of rotation, intertwine and finally create larger structures. Vortex merger was associated with the inverse energy and direct enstrophy cascades in two-dimensional and in quasigeostrophic turbulence.

Vortex merger was investigated first in 2D incompressible flows (Deem \& Zabusky (1978), Overman \& Zabusky (1982), Dritschel (1986), Melander, Zabusky \& McWilliams (1988)). A critical horizontal distance of 3.2 radii was found for the merger of piecewiseconstant vortices. More distant vortices co-rotate. Further studies addressed the case of unequal vortices (Melander, Zabusky \& McWilliams (1987), Dritschel \& Waugh (1992), Yasuda \& Flierl (1995), Yasuda \& Flierl (1997)). They determined how the critical merger distance varied with vortex size and strength. They also showed that in most cases, vortex merger was incomplete, or sometimes was replaced by vortex straining. In that instance, none of the fluid of one vortex merged into the other, but part of this fluid formed a filament which was expelled.

Vortex merger was also studied in 2-layer, rotating fluids. In that configuration, considerable effort was devoted to reconcile theory and laboratory experiments on the dependence of vortex merger on stratification (Griffiths \& Hopfinger (1987), Polvani, Zabusky \& Flierl (1989), Verron, Hopfinger \& McWilliams (1990), Valcke \& Verron (1993), Verron \& Valcke (1994)). It was then shown that the formation of baroclinic dipoles (the so-called hetons, see below) could counteract vortex merger.

The study of vortex merger was then extended to continuously stratified quasi-geostrophic flows, with vortices overlapping vertically (von Hardenberg at al. (2000), Dritschel (2002), Reinaud \& Dritschel (2002), Bambrey, Reinaud \& Dritschel (2007), Ozugurlu, Reinaud \& Dritschel (2008). As the vortices share some common horizontal layer, they can potentially merge, or more generally strongly interact. The interaction first depends on the vertical offset between the vortices. Vortices moderately offset in the vertical may merge for greater separation distances, see Reinaud \& Dritschel (2002). The interaction also depends on the respective aspect ratio and volume ratio between the vortices, the larger vortex dominating the interaction, see Bambrey, Reinaud \& Dritschel (2007). The potential-vorticity ratio between the two vortices is also important, and the interaction between vortices having significantly different strengths (volume-integrated potentialvorticity) generally results in the straining out of the "weak vortex" rather than the partial merger of the vortices, see Ozugurlu, Reinaud \& Dritschel (2008).

Interactions between two opposite-signed vortices are also common, and lead to vortex pairing, drift, or destruction via shearing (see Miyazaki, Yamamoto \& Fujishima (2003) and Reinaud \& Dritschel (2008)). In the latter, the authors showed that pairs of oppositesigned vortices can strongly interact. In particular they showed that the smaller vortex can break the larger one.

When like-signed vortices are vertically separated, their radial collapse and merger is called alignment (Polvani (1991), Viera (1995)). Alignment results in taller vortices and it is associated with the barotropisation of vortex structures in freely-decaying, geostrophic turbulence. Again dipolar effects can counteract vortex alignment (Correard and Carton (1999)).

We focus here on the stability and the nonlinear evolutions of two opposite-signed 
vortices, initially aligned vertically. These 'hetons' were introduced by Hogg \& Stommel (1985), to model baroclinic vortex pairs which can transport heat in the ocean. These early studies of hetons considered two point vortices in a 2-layer model. Flierl (1988) analysed the linear stability of finite-area baroclinic vortices with piecewise-constant potential vorticity, in a two-layer model. He found that the stability of these hetons depends on their radius-to-height aspect ratios. Tall hetons are stable. Increasing the radius-toheight aspect ratio favours baroclinic instability. Helfrich and Send (1988) modelled the nonlinear evolutions of such unstable vortices with contour dynamics. They observed a narrow domain of nonlinear stabilisation of perturbations at finite amplitude, for weakly unstable vortices. Sokolovskiy and Verron (2000) studied the nonlinear evolution of linearly unstable hetons and evidenced a noticeable tendency for pure baroclinic vortices to break into $l$ hetons where $l$ is the linearly most unstable wavenumber. On the contrary, the addition of a barotropic component could lead to nonlinear stabilisation of the perturbation, the perturbed vortex forming thus a baroclinic multipole. They also detailed the evolutions of initially tilted baroclinic vortices, of their propagation or breaking. Sokolovskiy (1997) investigated the stability of 3-layer axisymmetric vortices, with finite potential vorticity in the middle layer. Thus, he did not consider a heton with a vertical gap between the cyclone and the anticyclone. Moreover, to our knowledge, the stability of hetons has not yet been addressed within the framework of a continuous stratification.

How similar can heton stability properties be in continuously stratified and in layered model is the first objective of the present study; in particular, what is the influence of high baroclinic modes ? The second question is the influence of the aspect ratio of the heton and of the vertical gap between the two aligned vortices on heton stability. These questions are novel, in view of the aforementioned studies.

The paper is organised as follows. $§ 2$ presents the results for hetons in a continuouslystratified ocean. The governing equations are first reviewed then a linear stability analysis together with a few illustrations of nonlinear evolutions are proposed. $\S 3$ focuses on the same problem within the framework of a 3-layer model. Comparison between the two approaches and conclusions are drawn in $\S 4$.

\section{The continuously stratified quasi-geostrophic model}

\subsection{Formulation}

We present here the equations that govern a continuously stably-stratified and rapidlyrotating flow under the quasi-geostrophic (QG) approximation. For the sake of simplicity, we take both the buoyancy frequency $N$ and the Coriolis parameter $f$ constant, and we rescale the vertical direction $z$ by the ratio $N / f$. Note that at mid latitudes and for Central Waters, $N \sim 0.01 s^{-1}$ and $f \sim 0.0001 s^{-1}$. We neglect both diabatic heating and viscous diffusion. The governing equations read

$$
\begin{aligned}
\frac{\mathrm{d} q}{\mathrm{~d} t} & =0, \\
\Delta \psi & =q, \\
\boldsymbol{u}=(u, v, 0) & =-\boldsymbol{\nabla} \times \psi \mathbf{k} .
\end{aligned}
$$

where $q$ is the materially-conserved potential vorticity PV anomaly (hereinafter referred to as $\mathrm{PV}), \mathrm{d} / \mathrm{d} t=\partial / \partial t+\boldsymbol{u} \cdot \boldsymbol{\nabla}$ is the material derivative with $\boldsymbol{u}$ being the layerwise two-dimensional advecting velocity field, $\psi$ is the streamfunction, and $\Delta$ is the threedimensional Laplace's operator, see e.g. Vallis (2006) for further discussion.

We consider vortices of uniform PV. Such vortices are fully described by the shape of 
their boundary. Each vortex is discretised in the vertical by a set of $n_{c}$ horizontal PV contours, and each PV contour is discretised by $n_{p}$ nodes.

We first perform a linear stability analysis of cylindrical vortices aligned in the vertical direction. The vortices are taken to be axisymmetric in the horizontal, and therefore the overall PV distribution of the basic state is only function of $\rho$, the horizontal polar radius, yielding a steady state.

The basic state is a 'hot heton', consisting of a cyclonic vortex, with $q=2 \pi$, settled on the top of an identical anti-cyclonic vortex with $q=-2 \pi$, see Hogg \& Stommel (1985). Note that within the QG approximation, there is no asymmetry between cyclonic and anti-cyclonic vortex dynamical behaviour. Hence the reverse situation with an anticyclonic vortex on the top of a cyclonic vortex would give the same results. The time scale of the problem is set by taking $q= \pm 2 \pi$. For comparison, an isolated sphere of PV has a rotation period of $T=6 \pi /|q|$, while an infinite columnar vortex has a rotation period of $T=4 \pi /|q|$.

The linear stability analysis relies on deformation modes of the vortex boundaries. The analysis results in a standard eigenvalue problem in which the complex eigenvalues $\sigma=\sigma_{r}+i \sigma_{i}$ give the growth rate $\left(\sigma_{r}\right)$ and the frequency $\left(\sigma_{i}\right)$ of the modes. On the other hand, the eigenvectors give the spatial structure of the modes. Calculations are performed by explicit integration along contours in an infinite fluid domain. Details of the formulation may be found in Reinaud \& Dritschel (2002). In the linear stability analysis, the length scale of the problem is set by the total height of the heton, taken to be $\pi$.

We also illustrate the early stage of the nonlinear evolution of unstable hetons in a few cases. To that end, we use the Contour-Advective Semi-Lagrangian algorithm (CASL) developed by Dritschel \& Ambaum (1997). The QG equations are solved in a triply-periodic domain. To avoid a strong influence of the periodic images on the development of the flow, we confine the initial conditions in a $1^{3}$-box centred in the $(2 \pi)^{3}$ computational domain. During the late evolution of the heton, vortices may approach the boundaries and start to strongly interact with images, but we mainly focus here on the early destabilisation of the flow. The conversion grid which is used to convert the PV contours onto a fine gridded PV is $512^{3}$, while the inversion grid on which the Poisson's equation 2.2 is inverted is $128^{3}$.

\subsection{Results}

\subsubsection{Example case: $d / h_{v}=4 / 3$; linear stability analysis}

We consider first two cylindrical vortices of half-height $h_{v}=3 \pi / 16$ separated in the vertical by a gap of $d=\pi / 4$, hence $d / h_{v}=4 / 3$. The radius of the vortices is denoted $r$. The upper vortex has a uniform PV of $q=2 \pi$, while the lower one has a PV $q=-2 \pi$. The geometry is described in figure 1. Recall that the total height spanning the heton is $H=\pi$. Each vortex is spanned in the vertical by $n_{c}=45$ horizontal contours, while the gap corresponds to 30 layers. Each contour is discretised by $n_{p}=298$ nodes. The layer thickness is $\delta z=\pi / 120$. We perform the linear stability analysis of the heton for $1.8 \leqslant r / h_{v} \leqslant 10.0$ using an increment in $r / h_{v}$ of 0.2 . We have tested the accuracy of the method on the case $r / h_{v}=5$, by almost doublng the resolution, i.e. taking $n_{c}=90$ and $n_{p}=498$. The growth rates obtained are unchanged down to the five significant digits.

We first illustrate the growth rates and frequencies of the modes in figure 2(a). For $r / h_{v}<3.4$ the heton appears neutrally stable as $\sigma_{r}=0$ for all modes at the algorithm precision. One of these neutral modes has a frequency that decreases faster than the others as we approach $r / h_{v}=3.4$, as seen from figure 2 . There are in fact two modes with opposite frequencies that collapse to zero, only the positive one is displayed and we 


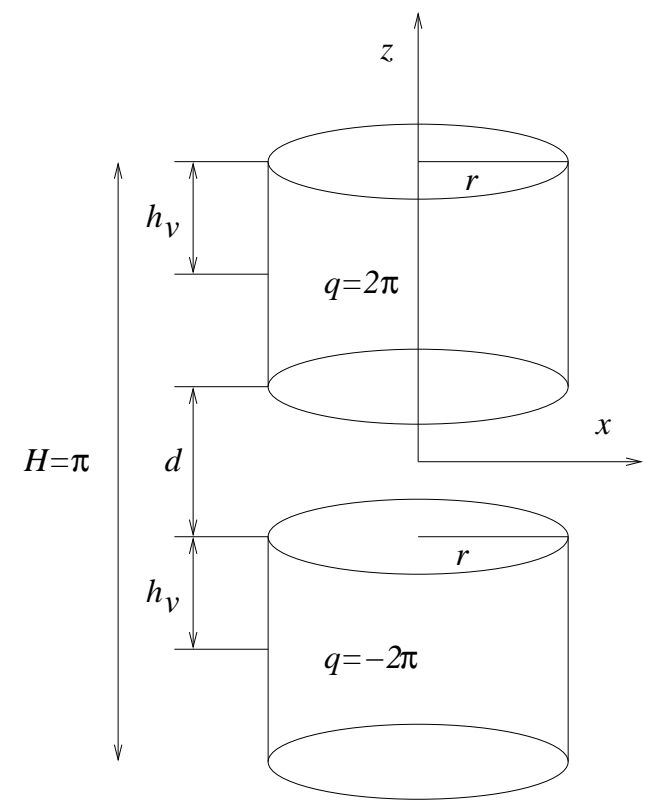

FIGURE 1. Geometry of the heton.

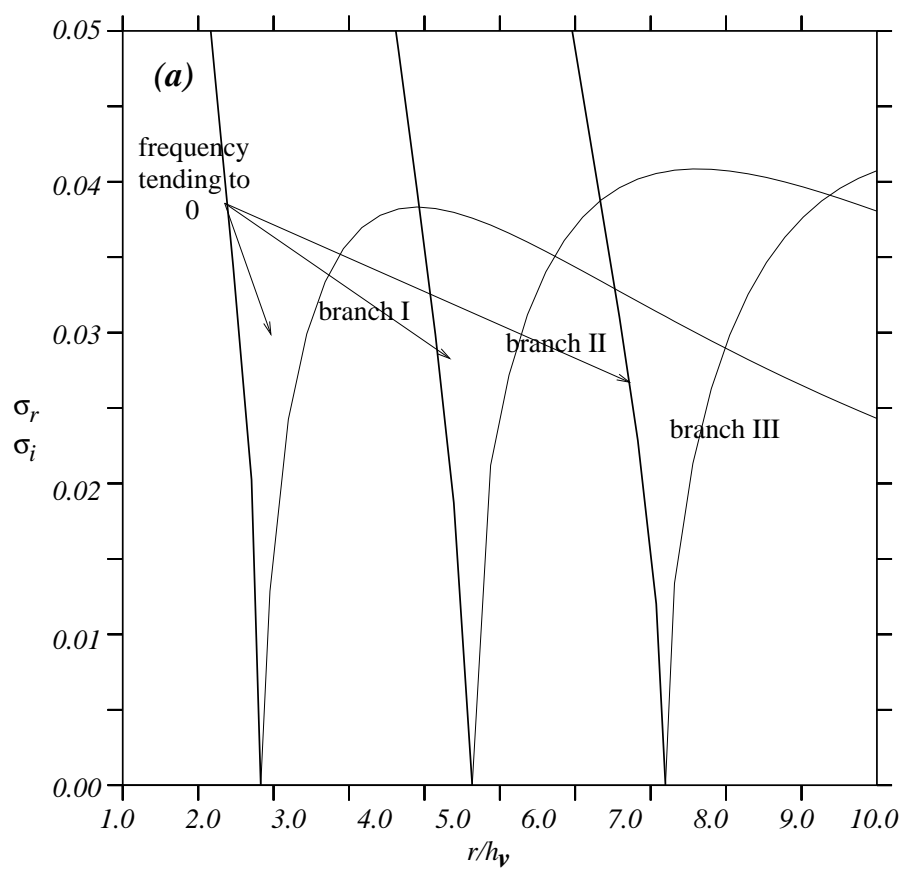

FiguRE 2. Growth rates $\sigma_{r}(+)$ and frequencies $\sigma_{i}(\Delta)$ of the modes versus the aspect ratio $r / h_{v}$ for cylindrical hetons $h_{v}=3 \pi / 16$ and $d=\pi / 4$.

will restrict attention to this one only. The frequency eventually vanishes and the mode becomes unstable (exchange-type instability). From $r / h_{v} \geqslant 3.4$, the unstable modes have zero frequency. The unstable mode is a double eigenvalue of the problem, due to the symmetry of the configuration. As the aspect ratio $r / h_{v}$ is increased, the growth rate 
6
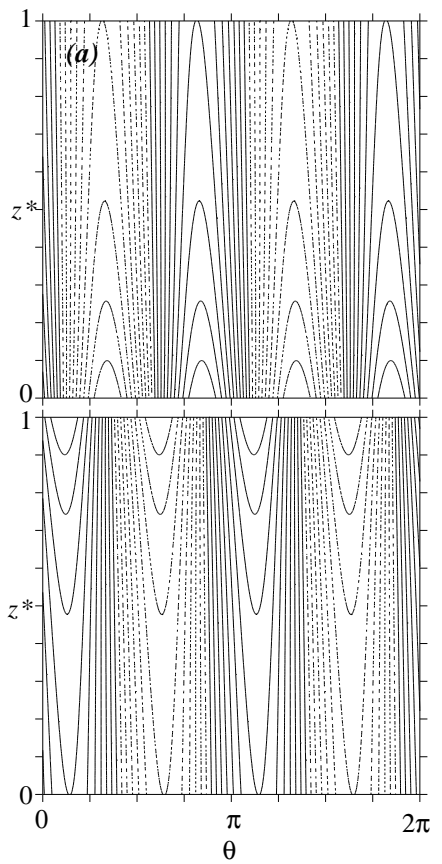

J. N. Reinaud and X. Carton
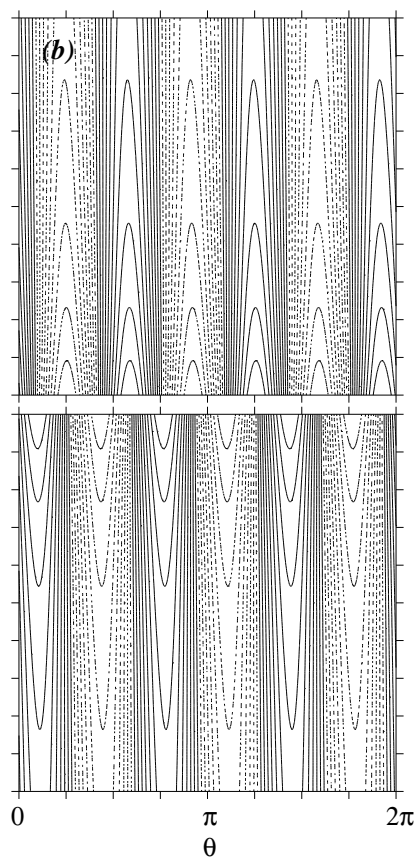
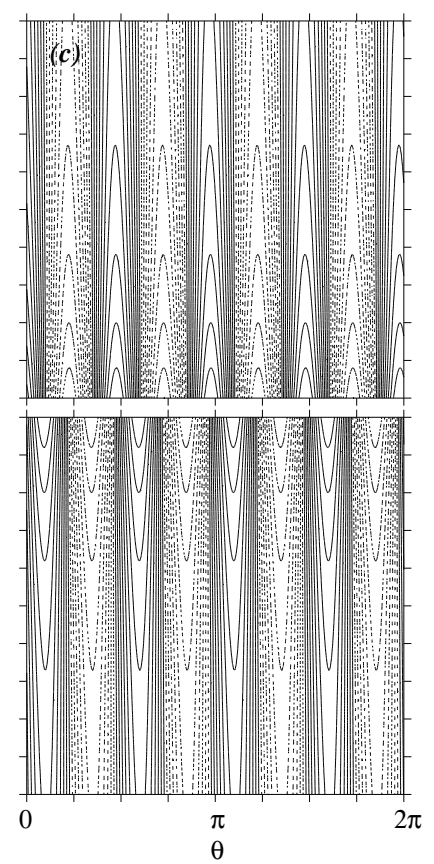

FiguRE 3. Spatial structure of the most unstable mode for $(a) r / h=5.0,(b) r / h=8.0$, (c) $r / h=10.0$. In each case the top figure represents the deformation of the top cyclonic vortex and the bottom figure the deformation of the bottom anti-cyclonic vortex. The vertical axis of each plot is the non-dimensional direction $z^{*}=\left(z-z_{i}\right) /\left(2 h_{v}\right)$, where $z_{i}$ corresponds to the vertical coordinate of the bottom of the vortex $i, i=1,2$. The horizontal axis is the azimuthal angle $0 \leqslant \theta \leqslant 2 \pi$. Solid contours correspond to negative deformation - inward deformation while dashed contours correspond to positive deformation - outward deformation.

of this unstable mode increases to reach a maximum $\sigma=0.03832$ for $r / h_{v}=5.0$. For $r / h_{v}>5.0$, the growth rates along this branch of unstable modes (branch $I$ ) decrease when increasing $r / h_{v}$. Meanwhile, a second branch (branch $I I$ ) of instability appears for $r / h_{v} \geqslant 5.8$. Qualitatively, this branch is similar to the previous one and stems from a mode whose frequency has collapsed to zero. The growth rate first increases up to $r / h_{v}=8.0$, where $\sigma_{r}=0.04085$ then decreases for increasing $r / h_{v}$. Modes from branch $I I$ become more unstable than modes from branch $I$ from $r / h_{v}=6.8$. A third branch appears (branch $I I I$ ) from $r / h_{v}=7.8$, and becomes the most unstable mode from $r / h_{v}=9.6$. We do not study the hetons for $r / h_{v}>10$. Such structures are arguably very rare in the oceans. Branch $I I I$ has not saturated yet by $r / h_{v}=10$ (i.e. has not reached its maximum growth rate). Finally a fourth branch starts at $r / h_{v}=10$.

We determine the spatial structure of the unstable modes, by analysing the eigenvalues. Branch $I$ corresponds to azimuthal wavenumber $l=2$, branch $I I$ to $l=3$ and branch $I I I$ to $l=4$. The sequence continues for larger $r / h_{v}$, with the fourth branch corresponding to $l=5$ and so on.

The spatial structure of the most unstable mode is illustrated in figure 3 for $r / h_{v}=$ 5,8 , and 10 . We verify that for the most unstable mode has an azimuthal number $l=2$ for $r / h_{v}=5, l=3$ for $r / h_{v}=8$, and $l=4$ for $r / h_{v}=10$. These deformation modes are generic to all cases studied. We see, as one expects, that the deformation is the largest at the bottom of the top vortex and the top of the bottom vortex, parts of the vortices which most strongly interact with one another. We also note that the deformation of 


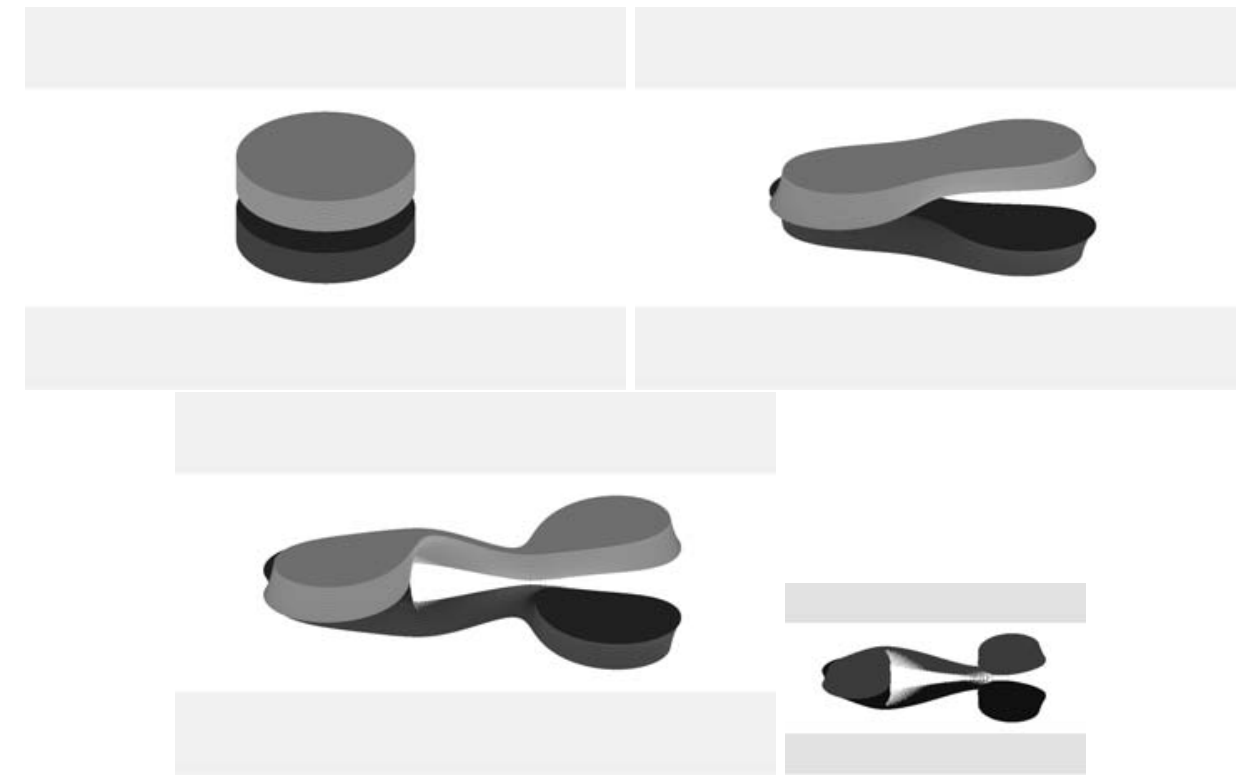

FiguRE 4. Nonlinear evolution of the heton with $r / h=5$. Times displayed are $t=0,81,87,89$. Vortices are viewed orthographically at an angle of $60^{\circ}$ from the vertical.

each PV contour for a given vortex is in phase, i.e. the deformation does not twist the vortex. However the deformation between the top and bottom vortices has a phase of $\pi /(2 l)$, hence for example, azimuthal angles corresponding to maximum or minimum deformation for the top vortex correspond to zero-deformation for the bottom vortex.

\subsubsection{Nonlinear evolutions of vortices in case: $d / h_{v}=4 / 3$}

We next illustrate the nonlinear evolution of the heton for the three examples detailed in the previous paragraph, namely $r / h_{v}=5,8$, and 10 with $d / h_{v}=4 / 3$.

In the first example $\left(r / h_{v}=5\right)$, the initial conditions are two cylindrical vortices centred respectively at $(0,0,-5 / 16)$ and $(0,0,5 / 16)$ and of radius $r=15 / 16$. The total height of the structure is 1 , so that the vortices initially fit in the $1^{3}$ box centred at the origin of the $(2 \pi)^{3}$ computational domain. Each vortex is discretised by 30 contours. The nonlinear evolution of the heton is shown in figure 4. In this example, as in the following ones, the flow is not explicitly disturbed. The perturbation builds up from the weak numerical noise. This noise is present at all spatial scales available, and we do not bias the outcome of the interaction by forcing a specific mode. The heton remains virtually not deformed until $t \simeq 65$. Then the azimuthal mode $l=2$ starts to develop. Note that in the case, according to the linear stability analysis, only mode $l=2$ is unstable, with a growth rate $\sigma_{r}=0.03832$. Both vortices deform quasi-elliptically and we recover a phase difference of about $\pi / 4$ between the deformation of the two vortices, in agreement with the linear stability analysis. Indeed, this $\pi / 4$ vertical phase shift maximises the growth of baroclinic instability (via a maximal conversion of available potential energy into eddy kinetic energy). As the flow develops it becomes slightly asymmetric. Each vortex further deform into dumbbell-shaped vortices, with poles increasingly moving away from the centre of the domain. Eventually the bridge of PV linking the poles of each vortex is stretched and breaks, and essentially 4 poles are generated, with filaments and debris remaining at the centre of he domain. Note that at the late evolution of the flow, vortices 


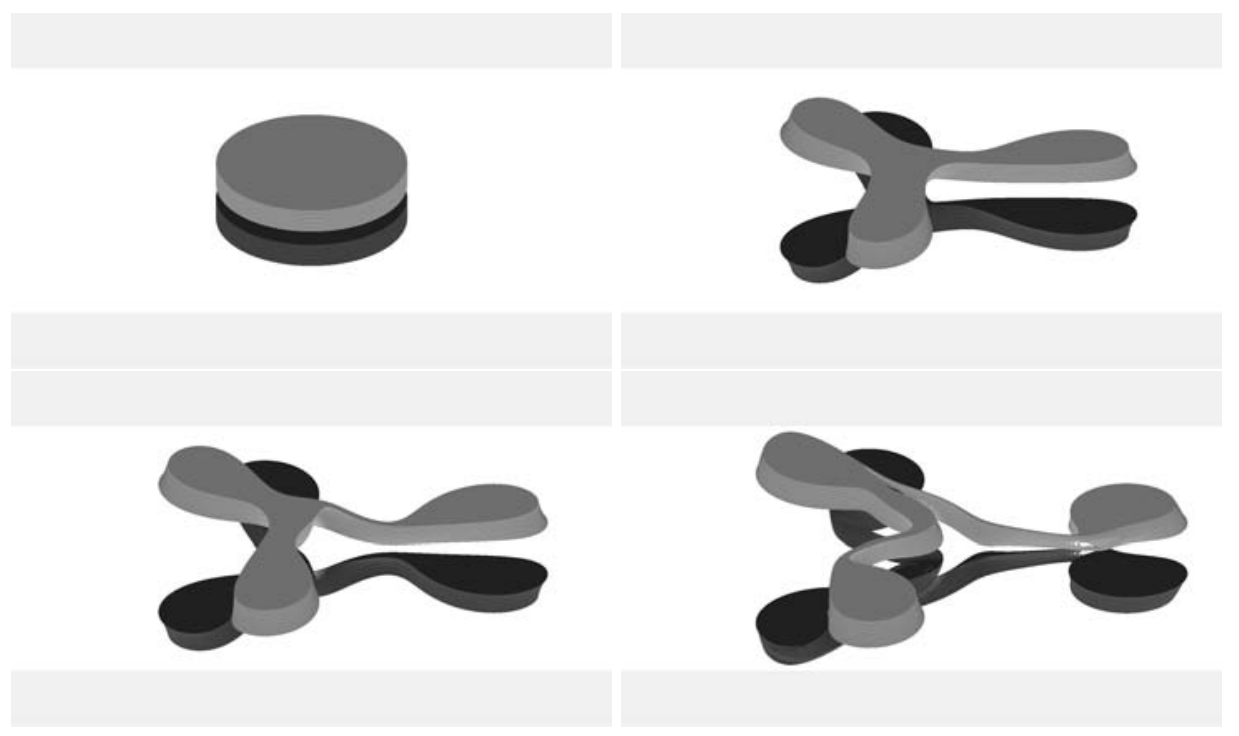

FiguRE 5. Nonlinear evolution of the heton with $r / h=8$. Time displayed are $t=0,61,64,68$. Vortices are viewed orthographically at an angle of $60^{\circ}$ from the vertical.
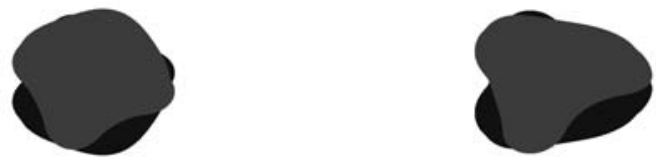

Figure 6 . Nonlinear evolution of the heton with $r / h=8$. Time displayed are $t=44$, and 51 . Vortices are viewed orthographically at an angle of $30^{\circ}$ from the vertical.

are very close to the boundary of the domain and the influence of the periodic images becomes important.

In the second example $\left(r / h_{v}=8\right)$, the two vortices are centred at $(0,0,-5 / 24)$ and $(0,0,5 / 24)$ at $t=0$, and they have unit radius. The total height of the heton is $2 / 3$, so the initial conditions fit in the $1^{3}$ box. Each vortex is discretised by 21 contours. As seen in the previous subsection, in this case the most unstable mode corresponds to $l=3$ with $\sigma_{r}=0.04085$. We can expect that this mode will dominate the evolution of the heton. However, we have to keep in mind that this mode is not the only unstable mode. Modes $l=2$, with $\sigma_{r}=0.03009$, and $l=4$, with $\sigma_{r}=0.02133$ also develop. The heton evolution is illustrated in figure 5 . It is shown that mode $l=3$ dominates the nonlinear evolution of the flow. Each cylindrical vortex reorganises into a tripolar vortex, and the poles move away from the centre of the domain, as clearly seen from $t=61$ in figure 5 . The poles are not equally distributed in the azimuthal direction, and this is due to the influence of the two other unstable modes which compete with the mode $l=3$. We also see a small phase between the two vortices which is consistent with the results of the linear stability analysis. 


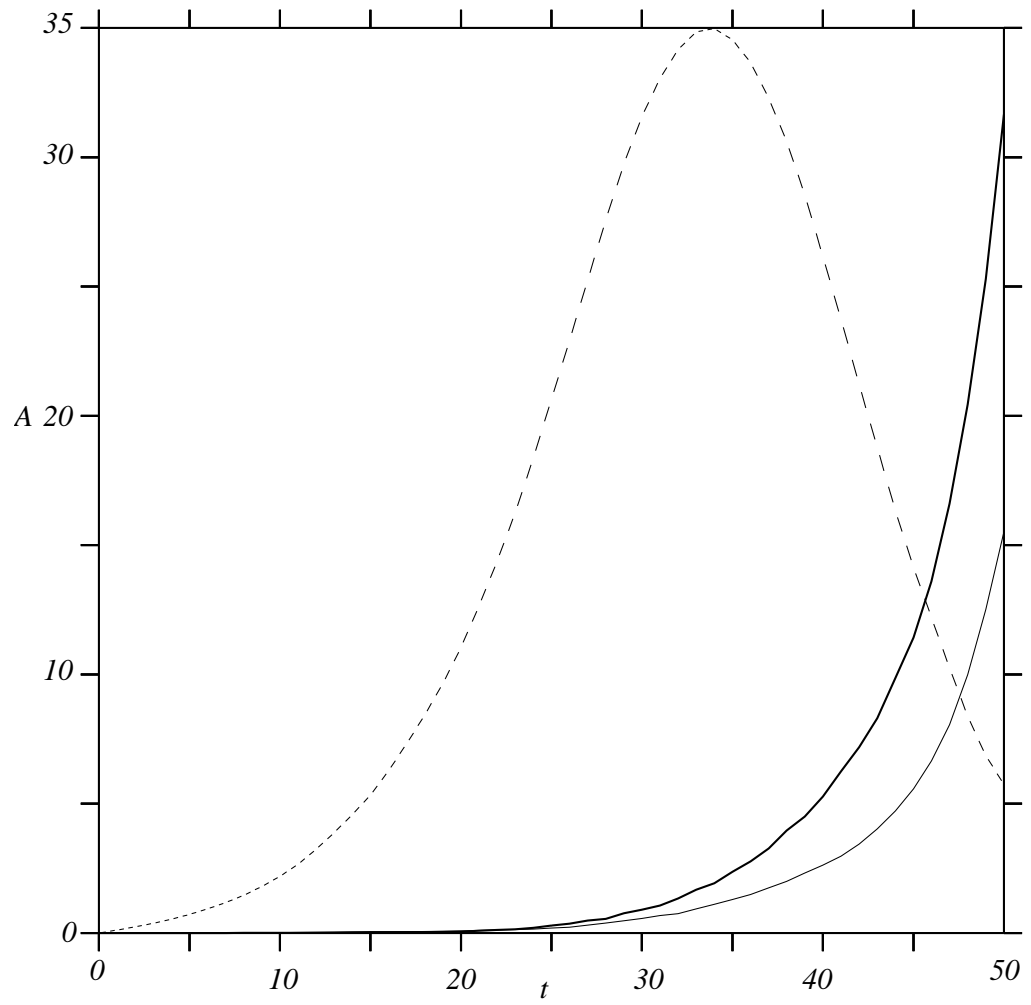

Figure 7 . Time evolution of the norm of the Fourier modes of the contour deformation for the azimuthal modes $l=2$ (thin solid line), $l=3$ (bold solid line) and $l=4$ (dashed line).

Eventually the heton breaks generating 6 main vortices with filaments and debris near the centre of the domain. In this case we see that the mode $l=3$ leads the long term evolution of the flow. However, interesting dynamics occurs at an early stage of the evolution. From $t=0$, the 3 unstable modes grow from the numerical noise. When the deformation becomes nonlinear, the most apparent mode is $l=4$, see figure 6 . Yet this mode saturates early and weak nonlinear effects stabilise this mode. As soon as the mode $l=4$ virtually disappears, the mode $l=3$ grows rapidly and dominates the late nonlinear stages of the flow.

To better judge the time evolution of the different modes of instability, we analyse the deformation of the PV contours bounding the hetons. More specifically, we first calculate for each contour the departure from the initial axisymmetric cylindrical shape. We then perform a Fourier transform, and sum over the number of contours the norm of the Fourier coefficients for the three unstable modes, $l=2$, 3, and 4 . Results are shown in figure 7 . Mode $l=4$ first grows rapidly and saturates at $t=34$. Modes $l=3$ and $l=2$ grow from an infinitesimal numerical noise and mode $l=3$ is the most amplified, yet the mode $l=2$ has a similar amplitude which explains the asymmetry in the later evolution of the heton.

The last example consists of two cylindrical vortices with $r / h_{v}=10$. Vortices are placed at $(0,0,-5 / 30)$ and $(0,0,5 / 30)$ and have unit radius. The total height of the heton is $16 / 30$, such that the initial conditions fit in the $1^{3}$ box. Here the vortices are unstable to 4 azimuthal modes. The most unstable mode is $l=4$, with $\sigma_{r}=0.04074$. The 3 other modes are $l=3$ with $\sigma_{r}=0.03805$, then $l=2$ with $\sigma_{r}=0.02431$, and finally $l=5$, with 


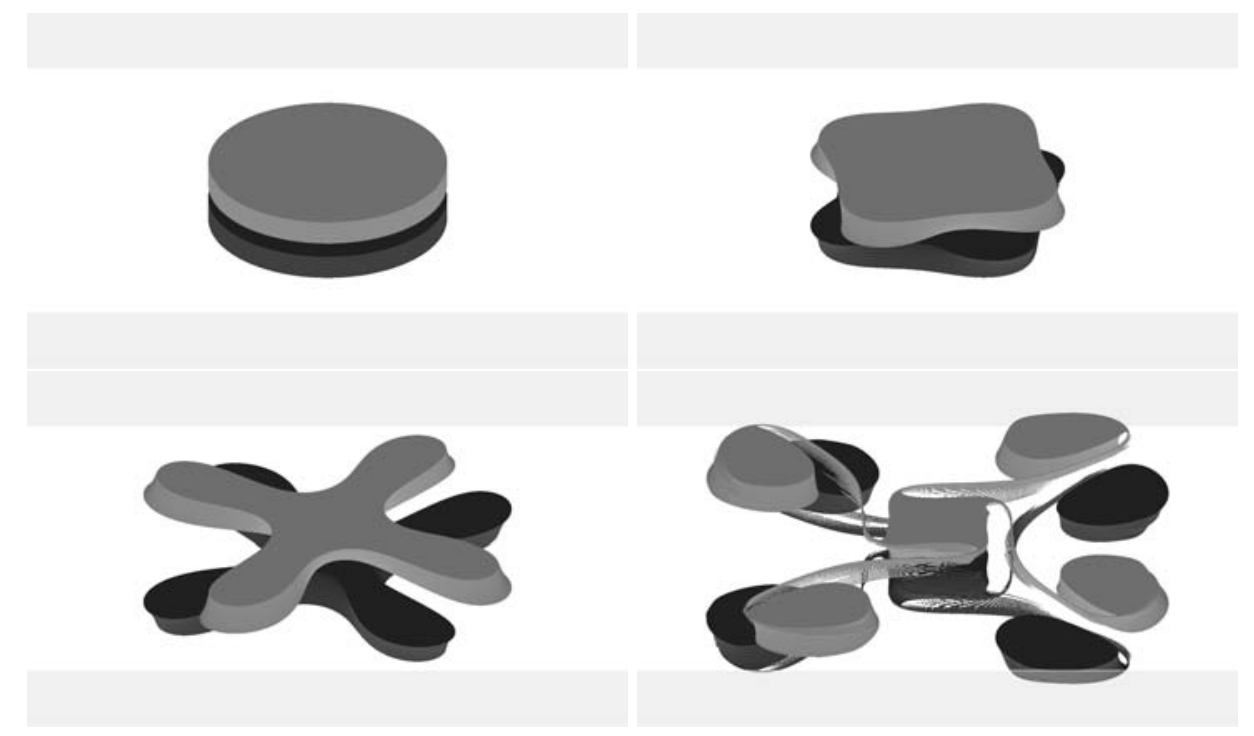

Figure 8. Nonlinear evolution of the heton with $r / h=10$. Time displayed are $t=0,18,29,41$. Vortices are viewed orthographically at an angle of $60^{\circ}$ from the vertical.

$\sigma_{r}=0.01654$. Each vortex is discretised by 16 contours. An illustration of the nonlinear evolution of the heton is proposed in figure 8 . From $t=0$, the mode $l=4$ dominates the evolution of the flow. The vortices first deform toward square-shaped vortices, then the corners move away from the centre. By $t=29$, each quadrupolar vortex has a fourblade propeller shape, and the deformation exhibits a phase between the two vortices of about $\pi / 8$ as predicted by the linear theory. The poles of the quadrupolar vortices later separate, generating four pairs of peripheral vortices. The two vortices break into 10 main vortices: four peripheral hetons and a central baroclinic vortex with square shape. A small amount of filaments is also generated during the interaction.

These examples of nonlinear evolution of unstable heton back the predictions of the linear theory, and they show that hetons are highly unstable structures on their own.

\subsubsection{Influence of the vertical gap $d$}

We next investigate the linear stability of the hetons over a broader parameter space. In particular, we consider the influence of the relative vertical gap $d / h_{v}$ on the stability property of the flow. We consider 26 values for the vertical gap, $0 \leqslant d / h_{v} \leqslant 2$ with an increment of $2 / 25$. For each value of $d / h_{v}$, we analyse 50 aspect ratios $r / h_{v}, 0.2 \leqslant r / h_{v} \leqslant$ 10 with an increment of 0.2 , that is we investigate the stability of 1300 individual hetonic structures. To this end, we decrease the resolution to limit the computational cost, while retaining high accuracy. Each vortex is spanned by $n_{c}=25$ contours and each contour is discretised by $n_{p}=198$ nodes. The previous subsection shows on a few examples that is the most unstable mode dominates the nonlinear evolution the heton. In this section, we analyse the azimuthal wavenumber of the most unstable mode as a function of $r / h_{v}$ and $d / h_{v}$.

This can be done in a straightforward manner. For each value of $d / h_{v}, r / h_{v}$ we select the mode which has the largest growth rate. Then we extract the part of the eigenvector for this mode corresponding to the deformation of the bottom contour of the cyclonic vortex (the vortex on the top). The number of changes of sign in the deformation perturbation equals twice the azimuthal mode. We gather this information for all values of 


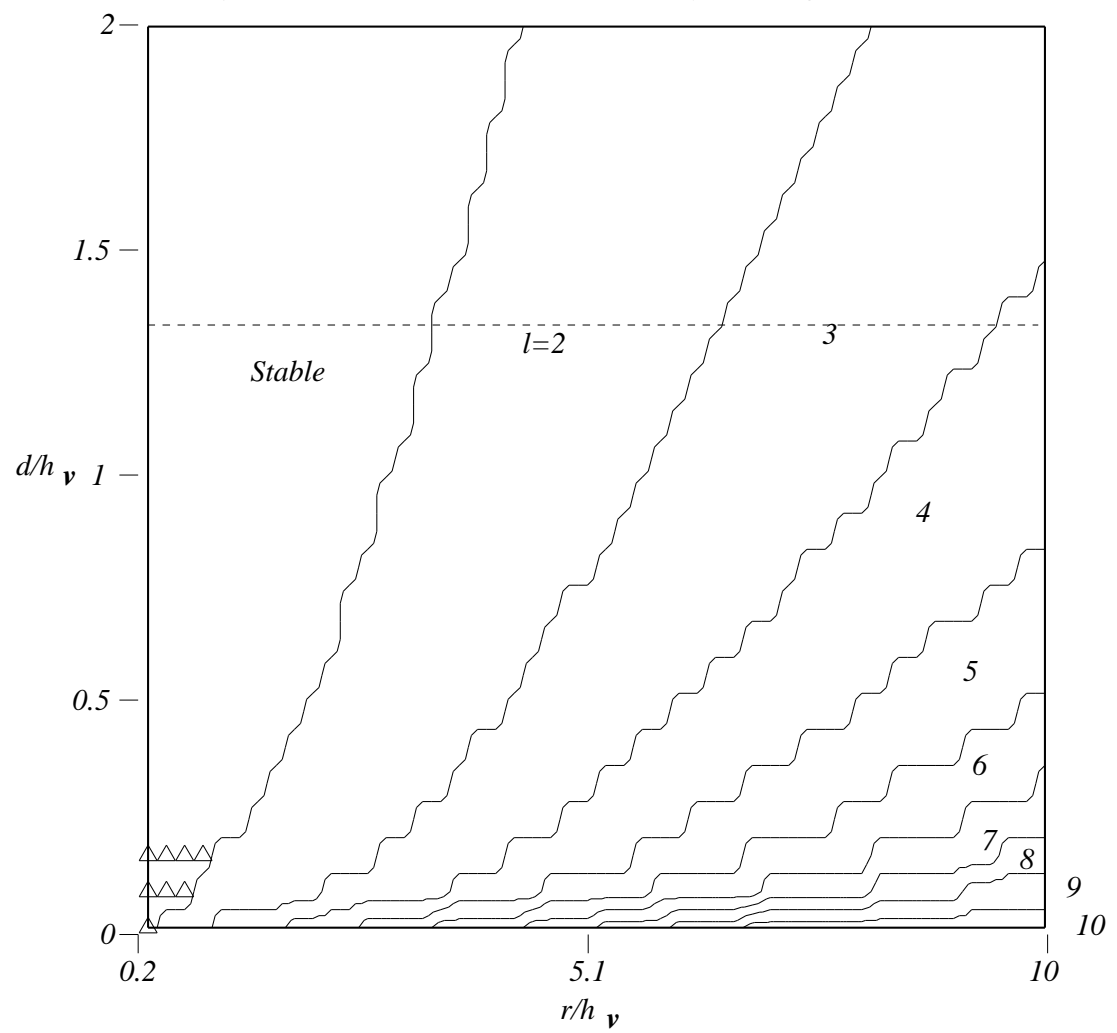

Figure 9. Most unstable modes in the parameter space $r / h_{v}, d / h_{v}$. The horizontal dashed-line indicates $d / h_{v}=4 / 3$, corresponding to the cases analysed in the previous section. The triangles indicate the region of unstable $l=1$ mode.

$d / h_{v}$ and $r / h_{v}$ and draw contours for fictitious intermediate values for the mode such as $1.5,2.5$ and so on, which delimits the different regimes of instability. Results are shown in figure 9. For each value of $d / h_{v}$, we recover (qualitatively) a similar behaviour as $r / h_{v}$ is varied. Increasing $r / h_{v}$ makes the heton more sensitive to perturbations with higher azimuthal wavenumber. However, there is a significant influence of the vertical gap, especially for $d / h_{v}$ small. For $d=0$, mode $l=2$ is the most unstable even when $r / h_{v}=0.4$, to be compared to $r / h_{v}=3.4$ for the example detailed in the previous section where $d / h=4 / 3$. For $r / h_{v}=10$, the most unstable mode is $l=10$ when $d=0$. Recall it was only $l=4$ for $d / h_{v}=4 / 3$. Even if the influence of the vertical offset decreases for larger values of $d / h$, it remains significant. We next plot the maximum growth rate (most unstable mode) in the $\left(r / h_{v}, d / h_{v}\right)$ parameter space in figure 10. The bottom contours correspond to $\sigma=0.1$. As $d / h_{v}$ is increased, the growth rate decreases as the mutual influence of the vortices weakens. The contour interval is $\mathrm{d} \sigma=0.01$. In figure 9 , the region to the left of the contour that delimits the region $l=2$, consists of the region where the hetons are neutrally stable. This is true but for a very small subregion where instability is observed. This region is only found for both $d / h_{v}$ and $r / h_{v}$ small, and covers a very small area in the parameter space and is indicated in figure 9 . This is confirmed in figure 10. The instability comes from the existence of an unstable baroclinic mode $l=1$ in this part of the parameter space. This mode is found to be dominant for $d=0$ and $r / h_{v}=0.2, d / h_{v}=2 / 25$ and $0.2 \leqslant r / h_{v} \leqslant 0.6$, and finally $d / h_{v}=4 / 25$ and 


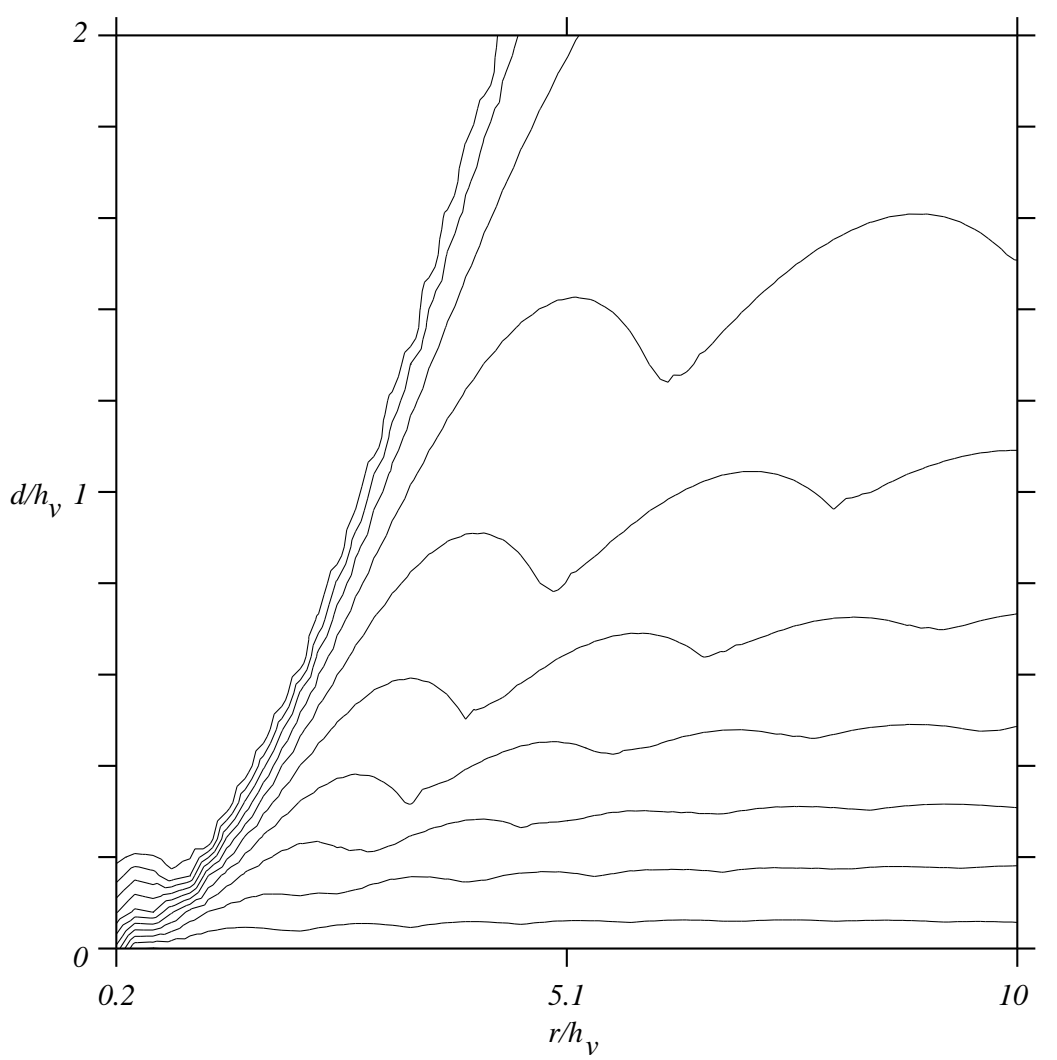

Figure 10. Maximum growth rate in the parameter space $r / h_{v}, d / h_{v}$. The contour interval $\mathrm{d} \sigma=0.01$. The bottom contour represents $\sigma=0.1$.

$0.2 \leqslant r / h_{v} \leqslant 0.8$. In all other cases in this region, there is no dominant, unstable $l=1$ mode.

Figure 11 shows the spatial deformation of the bottom vortex for the most unstable mode for $0 \leqslant d / h_{v} \leqslant 4 / 25$ and $0.2 \leqslant r / h_{v} \leqslant 0.8$. For the values of $d / h_{v}$ and $r / h_{v}$ mentioned in the previous paragraph we see that the mode has azimuthal wave number $l=1$. For a given PV contour, a $l=1$ disturbance can be seen as a shift of the contour in the horizontal direction. We see that the phase and magnitude of the perturbation along the vortex is $z$-dependent, that is, the perturbation is baroclinic. The unstable perturbation twists and bends the vortex. From the strong variation in the amplitude of the deformation as a function of the $z$, it is likely that only the top of the lower vortex and the bottom of the upper one can strongly interact. There is some similarity between what is observed in this situation and the tall-column instability discussed by Dritschel and de la Torre Juárez (1996) even if the situation is different here from the case investigated by the authors. This baroclinic instability affects tall vortices submitted to a strong deformation field due to the presence of a nearby vortex (the second vortex of the heton).

We finally illustrate this instability by a numerical experiment. The lower anti-cyclonic vortex is placed at $(0,0-0.525)$ and the upper cyclonic vortex at $(0,0,0.525)$. The radius of the vortices is $r=0.1$, and $r / h_{v}=0.2$. In this part of the parameter space only the mode $l=1$ is unstable. The flow evolution is illustrated in figure 12 . As seen from the figure, the vortices slightly bend near their edges. The bending motion is oscillatory with 


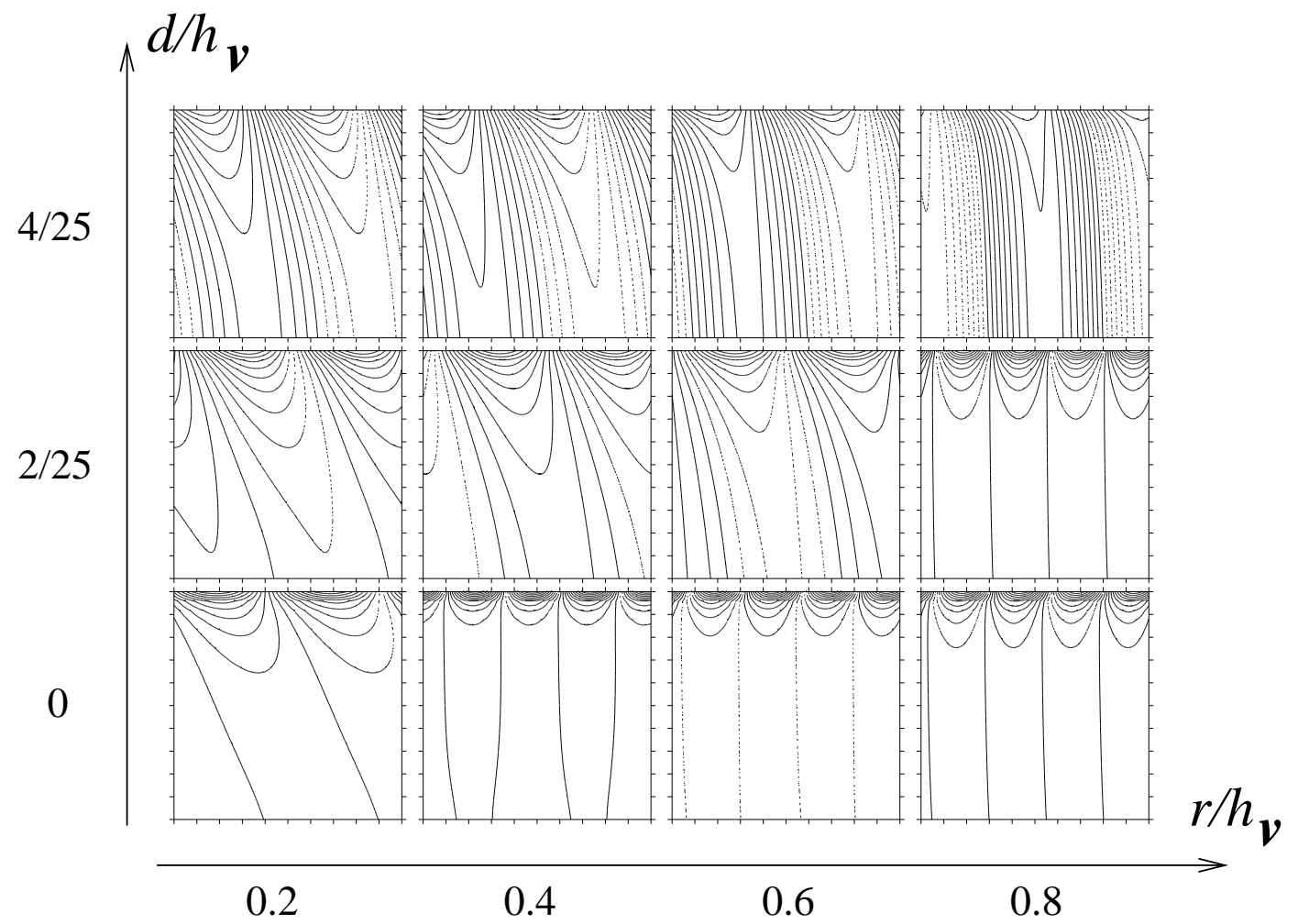

Figure 11. Spatial structure of the unstable baroclinic $l=1$ mode.

a frequency of $T \simeq 30$, i.e. 15 vortex turnovers. A few filaments are generated, and the vortex tips misalign. This induces a slow translation motion on the heton. However, this interaction remains weak, as the two vortices remain robust and do not break. The average translation velocity of the heton is about $5 * 10^{-3}$. Recall that the heton horizontal radius is 0.1 and its $\mathrm{PV}$ is $|2 \pi|$.

\section{Heton stability in the three-layer quasi-geostrophic model}

\subsection{Model equations}

In the QG layered model, potential vorticity is conserved in each layer under adiabatic, unforced and inviscid conditions

$$
\frac{\mathrm{d} q_{j}}{\mathrm{~d} t}=0
$$

with $j=1,2,3$ representing the upper, mid and lower layers.

Layerwise potential vorticity is written

$$
\begin{gathered}
q_{1}=\nabla^{2} \psi_{1}+F_{1}\left(\psi_{2}-\psi_{1}\right), \\
q_{2}=\nabla^{2} \psi_{2}+F_{2}\left(\psi_{1}-\psi_{2}\right)+F_{3}\left(\psi_{3}-\psi_{2}\right), \\
q_{3}=\nabla^{2} \psi_{3}+F_{4}\left(\psi_{2}-\psi_{3}\right) .
\end{gathered}
$$




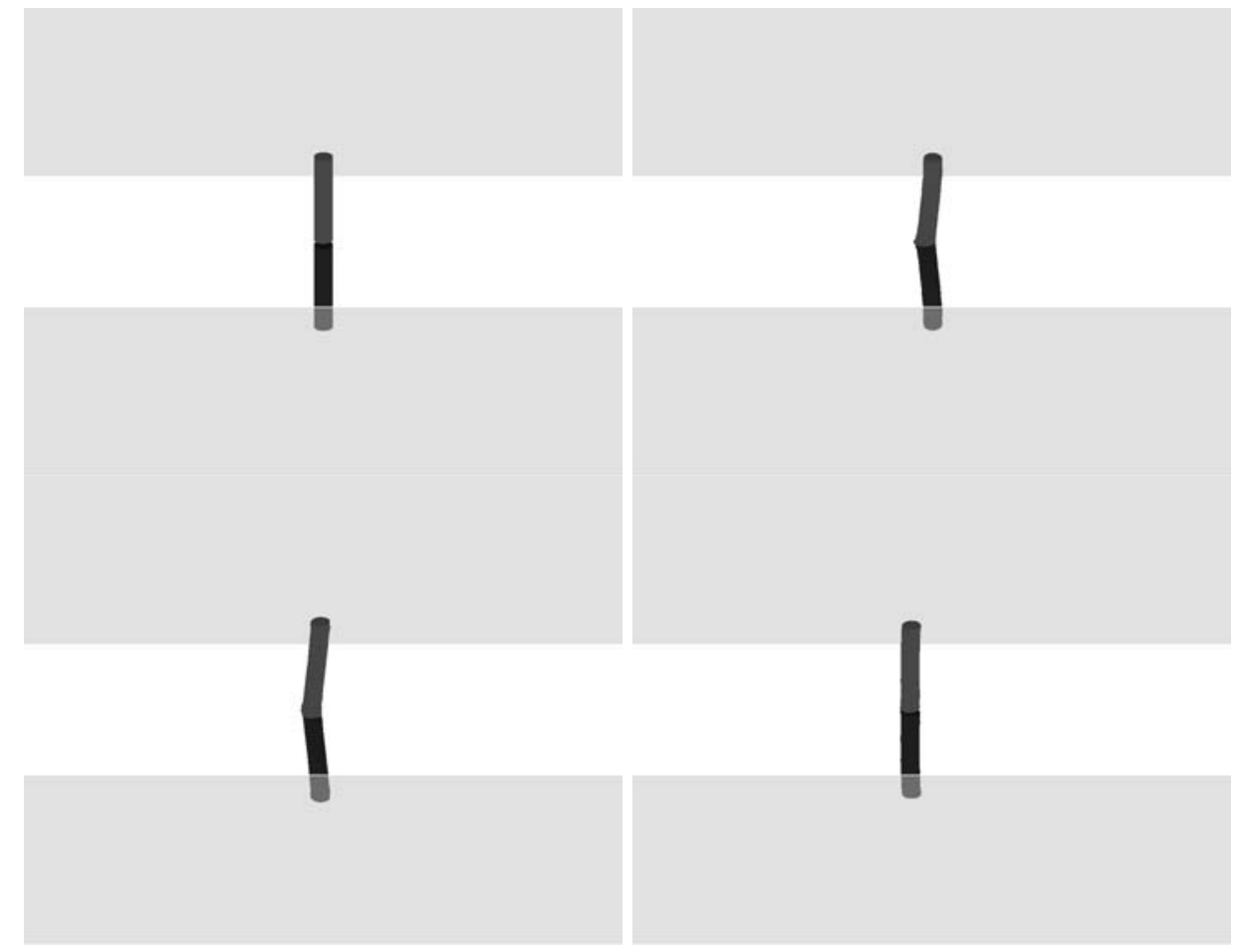

Figure 12. Nonlinear evolution of the heton with $r / h=0.2, d / h=0.1$. Time displayed are $t=0,80,90,120$. Vortices are viewed orthographically at an angle of $60^{\circ}$ from the vertical.

The linear stratification operator is represented here by matrix

$$
F=\left(\begin{array}{ccc}
-F_{1} & F_{1} & 0 \\
F_{2} & -F_{2}-F_{3} & F_{3} \\
0 & F_{4} & -F_{4}
\end{array}\right),
$$

where $F_{1}=f_{0}^{2} /\left(g_{1}^{\prime} H_{1}\right), F_{2}=f_{0}^{2} /\left(g_{1}^{\prime} H_{2}\right), F_{3}=f_{0}^{2} /\left(g_{2}^{\prime} H_{2}\right), F_{4}=f_{0}^{2} /\left(g_{2}^{\prime} H_{3}\right)$. The reduced gravities are $g_{j}^{\prime}$ and layer thicknesses at rest are $H_{j}$. For simplicity of the analytical calculations we assume that the total depth is unity in the 3-layer model, so that $H_{1}=$ $H_{3}=\left(1-H_{2}\right) / 2$. This choice does not prevent comparison with the continuously stratified model of section 2, since we can convert layer thicknesses into $d$ and $h_{v}$ via $d=\pi H_{2}$ and $h_{v}=\pi H_{1} / 2$. The calculation of vertical modes is detailed in appendices $\mathrm{A}$ and $\mathrm{B}$.

\subsection{Linear stability of the three-layer vortices}

The linear (normal-mode) stability of the columnar vortex with radius $r$ and layerwise $\mathrm{PV} q_{j}\left(q_{1}=2 \pi, q_{2}=0, q_{3}=-2 \pi\right)$ is considered. As shown in the appendices, the normal mode stability equation is for each $\mathrm{PV}$ front deviation

$$
L_{j k} \eta_{k}=c \eta_{j} .
$$

Here $L$ is the linear operator describing the linear dynamics of the perturbed vortex boundary (see appendix B), $\eta_{j}$ is the magnitude of the deviation of the vortex boundary from a circular state in layer $j$ and $c$ is a complex number providing the rotation rate $\operatorname{Re}(c)$ and the growth rate $\sigma_{r}=l \operatorname{Im}(c)$ of the perturbation. These growth rates are computed for various values of the parameters.

Figure 13 presents the maximum growth rates over $l=1-10$ in the $r / h_{v}, d / h_{v}$ plane, 

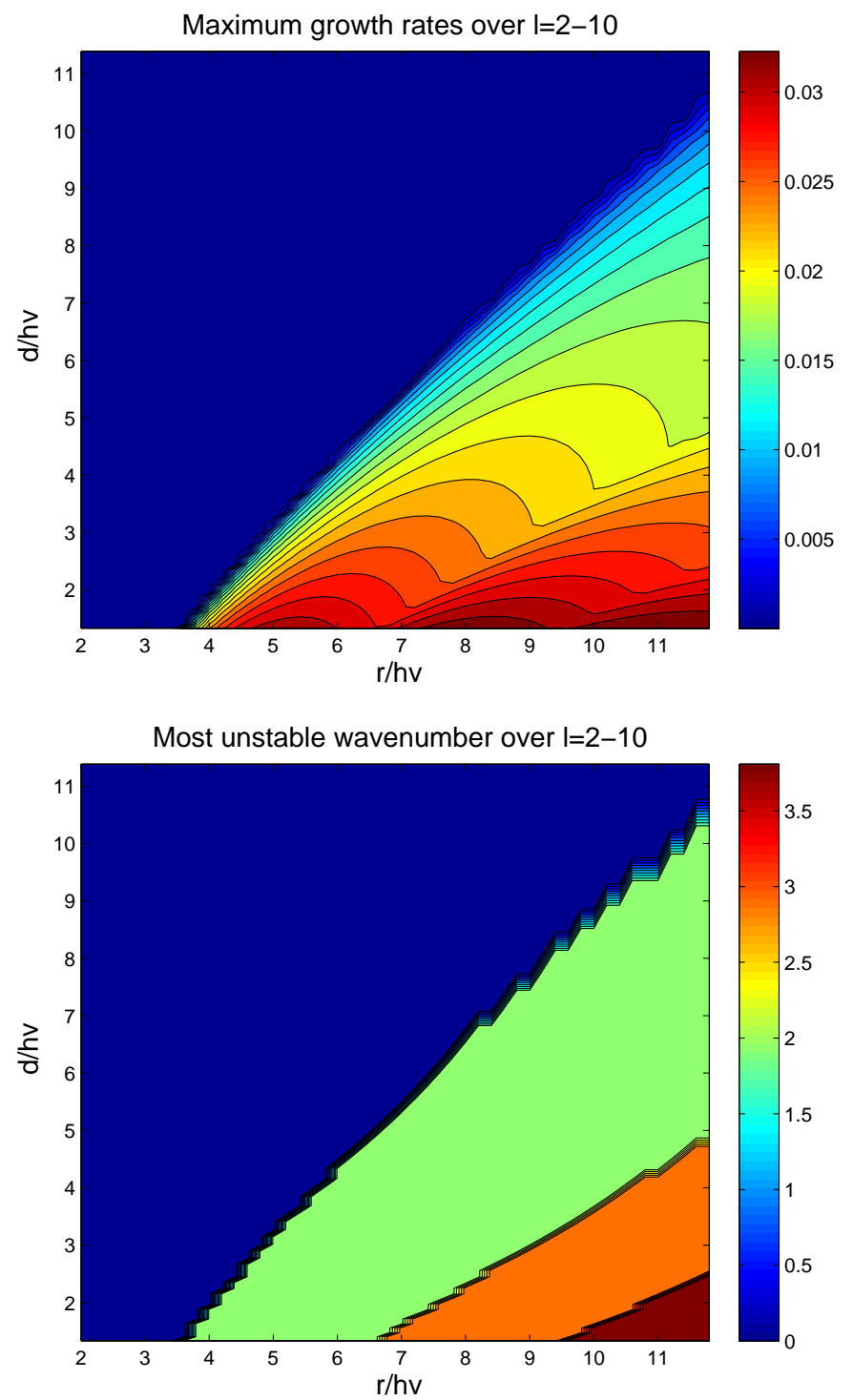

FigURE 13. Maximum growth rates and most unstable waves of normal-mode perturbations on the three-layer heton, in the $r / h_{v}, d / h_{v}$ plane. In the lower panel, vortices are stable in the blue region, most unstable to wavenumber $l=2$ in the green region, to $l=3$ in the orange strip and to $l=4$ in the dark red triangle.

in the three-layer model. Firstly, it evidences that mode $l=1$ is always stable for equal upper and lower layer thicknesses in the three-layer model (because stratification can be computed only for $d / h_{v} \geqslant 4 / 3$ in this case). Very shallow intermediate layers cannot be represented under these conditions and therefore the asymmetric mode $l=1$ found in the previous section cannot be attained. Secondly, this figure evidences the existence of successively dominant wavenumbers (here $l=2,3,4)$. Growth rates decrease for each wavenumber as $d / h_{v}$ increases because the interaction between the two active PV poles becomes weaker then. Higher modes $(l \geqslant 5)$ are not linearly unstable in this region of parameter space. 


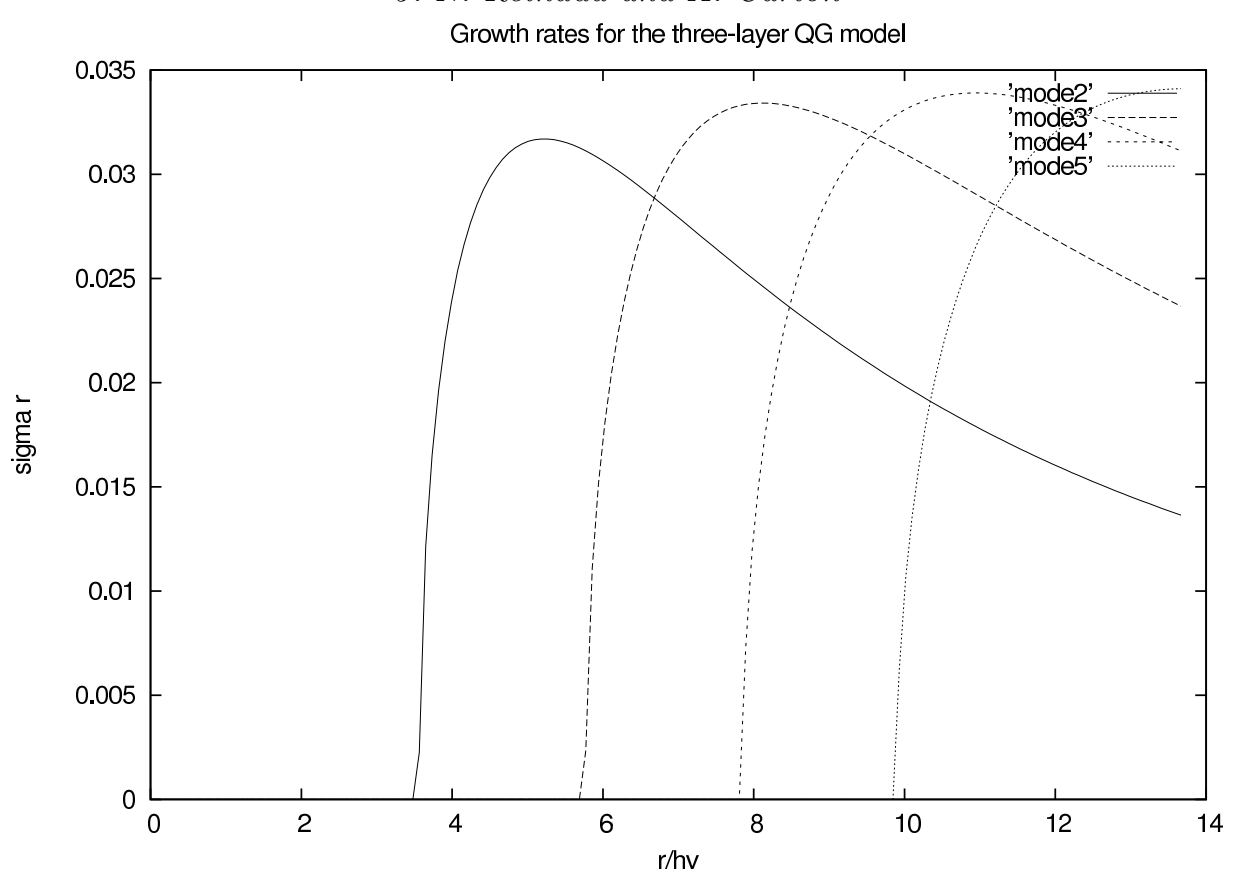

Figure 14. Normal mode instability of the three-layer heton (with zero PV in the intermediate layer), for $h_{v}=3 \pi / 16$ and $d=\pi / 4$, and for azimuthal modes from 2 to 5 .

Figure 14 is a section of figure 13 at $d / h_{v}=4 / 3$. It provides the growth rates of azimuthal modes $l=2$ to 5 . It can be compared to figure 2 (a) in the continuously stratified model.

Modes $l=2,3,4,5$ become unstable near critical radii $r / h_{v}=3.4,5.8,7.8,9.6$ as in the continuously stratified model. The values reached by the growth rates are comparable in the two models $\left(\sigma_{r} \sim 0.035\right)$ slightly increasing with the mode number. Growth rates peak at $r / h_{v} \sim 5.0,8.0,9.5$ for $l=2,3,4$ again as for continuous stratification. In the three-layer model, the structure of the perturbation is uniform vertically in each layer

Growth rates in the three-layer model are much smaller than in the two-layer model (by a factor $1 / 2$, results not shown here), since the two PV poles are separated here by a layer without PV anomaly which "shields" their interaction. Calling unscaled critical radii, the radii not divided by $h_{v}$, we note that these radii are comparable between the 2-layer and 3-layer models, though slightly larger in the 3-layer model. The agreement of our linear stability analysis in the two-layer case, with the Flierl (1988), Helfrich and Send (1988) and Sokolovskiy and Verron (2000) results, validates our procedure.

\subsection{Nonlinear evolution of the heton with zero $P V$ in layer 2}

The nonlinear evolutions of the hetons are computed in a pseudo-spectral Eulerian model which solves the equation

$$
\partial_{t} q_{j}+J\left(\psi_{j}, q_{j}\right)=\nu_{4} \nabla^{6} \psi_{j}
$$

The right-hand side is a numerical artifact which suppresses small scale features due to enstrophy cascade, but which does not alter the physical outcome of the simulation. The horizontal resolution of the model is $256 \times 256$. Low biharmonic viscosity was used $\left(\nu_{4}=5 \times 10^{-8}\right.$ in the $8 \pi \times 8 \pi$ domain and $\nu_{4}=2 \times 10^{-7}$ in the $16 \pi \times 16 \pi$ domain $)$. The baroclinic circular vortices are used as initial conditions of the model, with several types of perturbations to allow the most unstable mode to develop. 


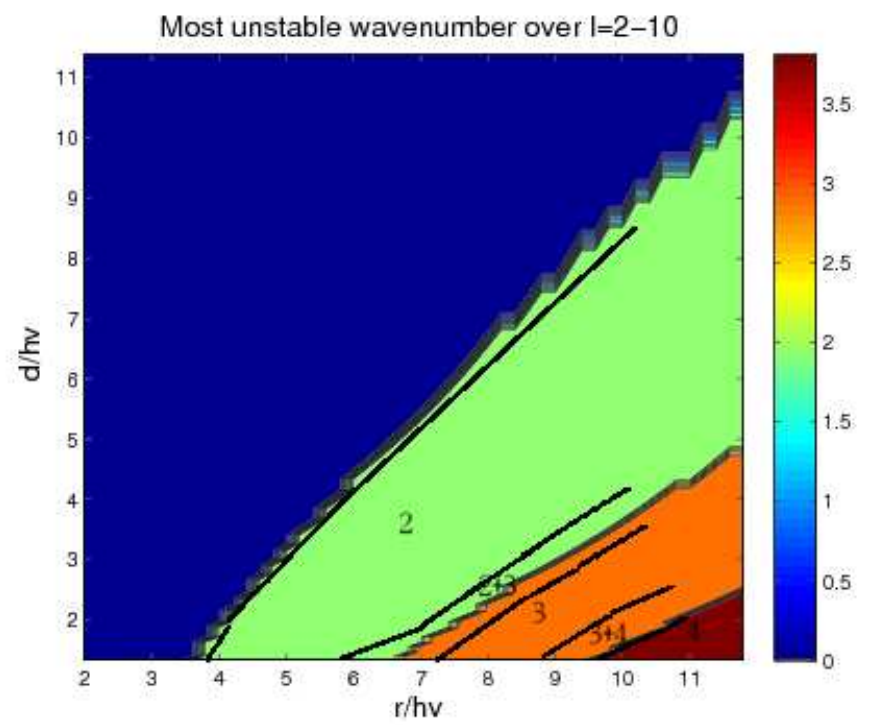

FiguRE 15. Nonlinear instability of the three-layer heton in the $\left(d / h_{v}, r / h_{v}\right)$ plane. The initial perturbation is a white noise in vorticity.

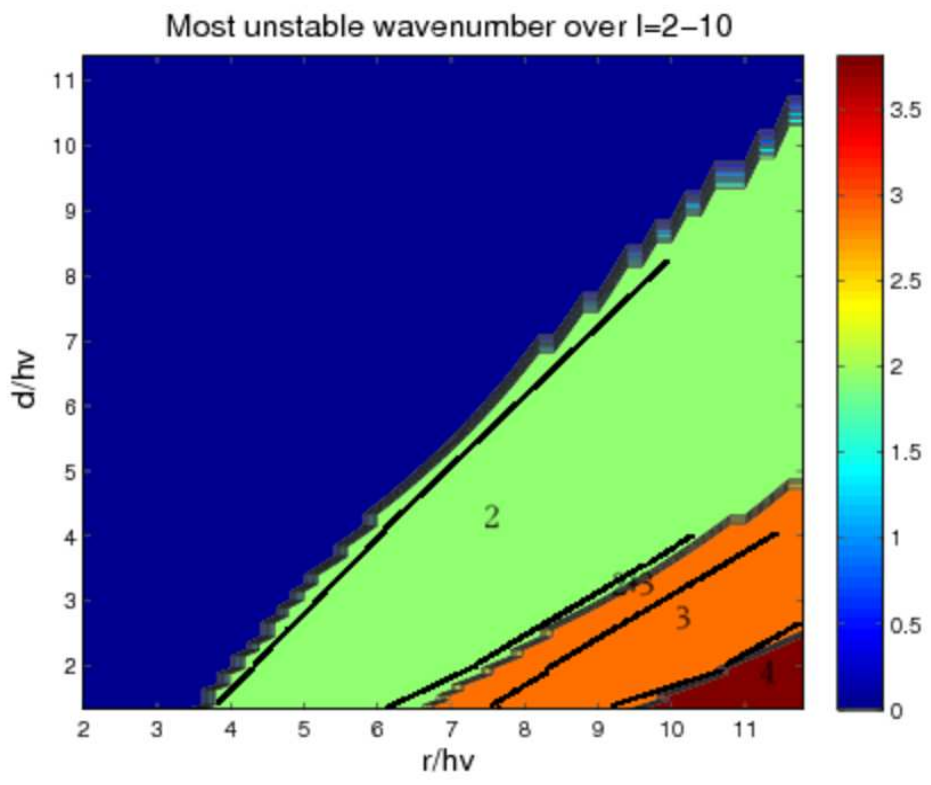

FIgURE 16. Nonlinear instability of the three-layer heton in the $\left(d / h_{v}, r / h_{v}\right)$ plane. The initial perturbation is a sum of $l=2 \mathrm{~m} l=3$ and $l=4$ modes, with equal amplitudes. $(2+3)$ indicate regimes where the vortex will either break into two dipoles with an asymmetric influence of mode 3 or conversely (the same holds for $(3+4)$.

Firstly, we checked the similarity of growth rates in the linear calculation and during the linear stage of nonlinear simulations (maximum growth rates over the linear period). We chose $d / h_{v}=4 / 3$ and values of $r / h_{v}$ near the peak instability of each mode. For $r / h_{v}=4.24, l=2$, the relative difference between linear and nonlinear growth rates 

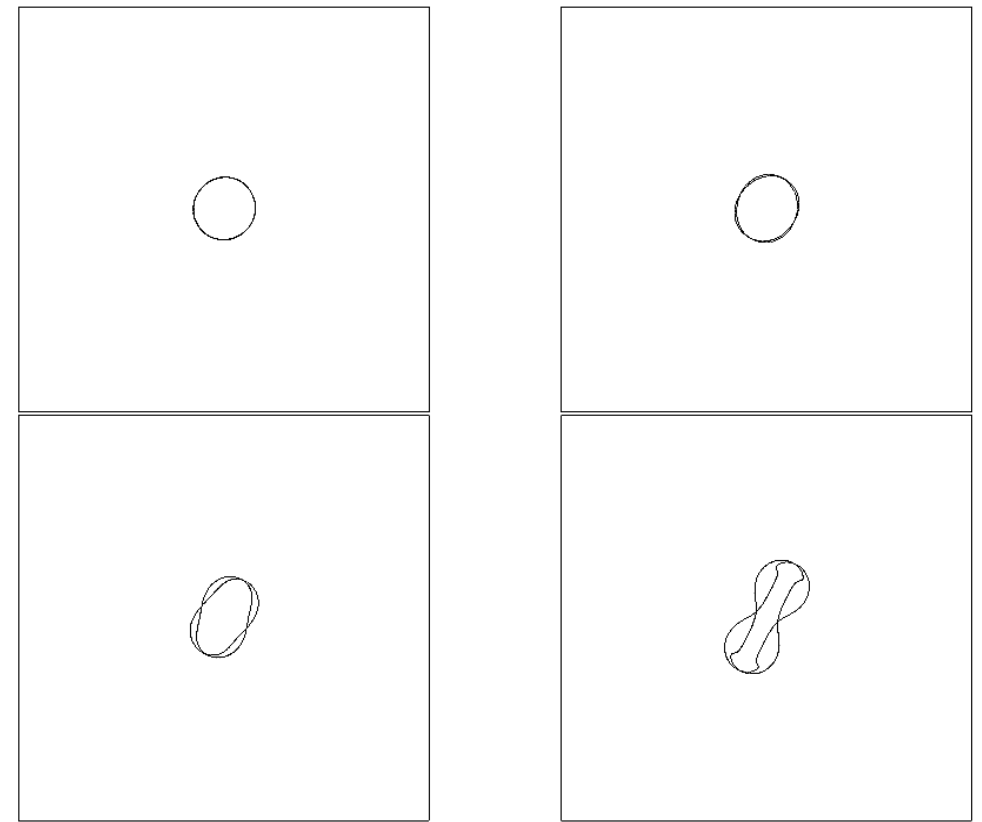

FIGURE 17. Horizontal maps of potential vorticity in layers 1 and 3 superimposed (contour $q_{j}= \pm 2 \pi$ ) for $r / h_{v}=5.1, d / h_{v}=4 / 3$, with initial superposition of equal amplitude mode 2 to 4 perturbations. Times shown range from $t=24$ to $t=48$ with increment $\delta t=8$, from left to right, and from top to bottom.

was $4 \%$; for $r / h_{v}=7.64, l=3$, the relative error was $5 \%$, and for $r / h_{v}=11.03, l=4$, the difference was $3 \%$. These figures were obtained in the largest domain and for a pure $l$-mode perturbation initially.

Secondly, we ran many numerical simulations of the nonlinear model, either with a white-noise perturbation in vorticity, or with an initial perturbation having an equal amplitude on modes $l=2,3,4$. Figures 16 and 15 show the regime diagram for these two cases, in the $\left(d / h_{v}, r / h_{v}\right)$ plane, superimposed on the linearly most unstable wave diagram.

The two plots clearly show that the initial baroclinic vortex breaks into $l$ peripheral hetons, drifting radially away from the centre. The value of $l$ obtained in the nonlinear model is comparable to the dominant linear mode, when the growth rate of this mode is at least $20 \%$ larger than those of the other modes (in particular for $l=3$ ). In the regions of the plane where the growth rates of modes $l$ and $l+1$ are comparable, the outcome of the nonlinear model is less predictable : the vortex will break into either $l$ hetons with an asymmetry on mode $l+1$ or conversely . This is clearly the case at the boundaries of the domain of mode $l=3$, and for an initial white-noise perturbation. In this case, the initial vortex breaks into 2 or 3 asymmetric hetons (on the left-hand side of the domain) and 3 or 4 asymmetric hetons (on the right-hand side of the domain).

Mode superposition is slightly reduced when the three modes $l=2,3,4$ are initialised at equal amplitude, compared with the white-noise perturbation. An explanation for this is that the amplitude of the most unstable mode is not controlled in a white noise perturbation (and therefore, can be smaller than that of other modes).

Mode superposition (in particular involving $l=3$ ) is also reduced when simulations are performed in a large domain. Since the initial perturbation is only 1/10000 of the mean 

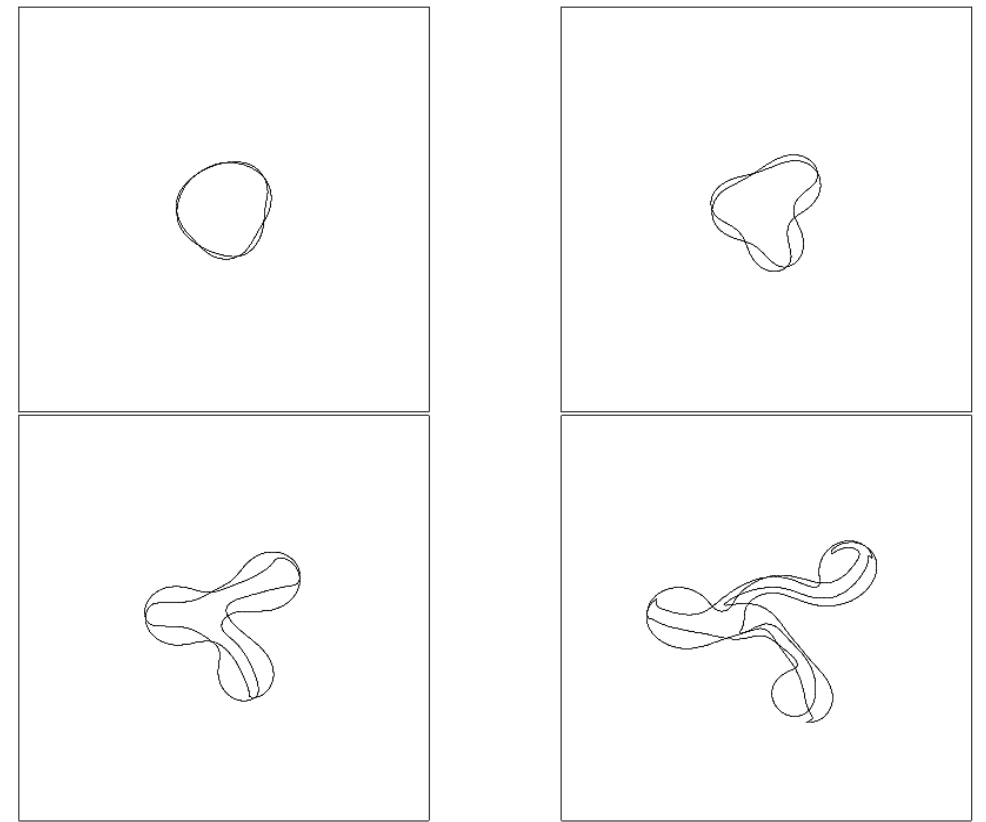

FIGURE 18. Horizontal maps of potential vorticity in layers 1 and 3 superimposed (contour $q_{j}= \pm 2 \pi$ ) for $r / h_{v}=8.5, d / h_{v}=4 / 3$, with initial superposition of equal amplitude mode 2 to 4 perturbations. Times shown range from $t=24$ to $t=48$ with increment $\delta t=8$, from left to right, and from top to bottom.
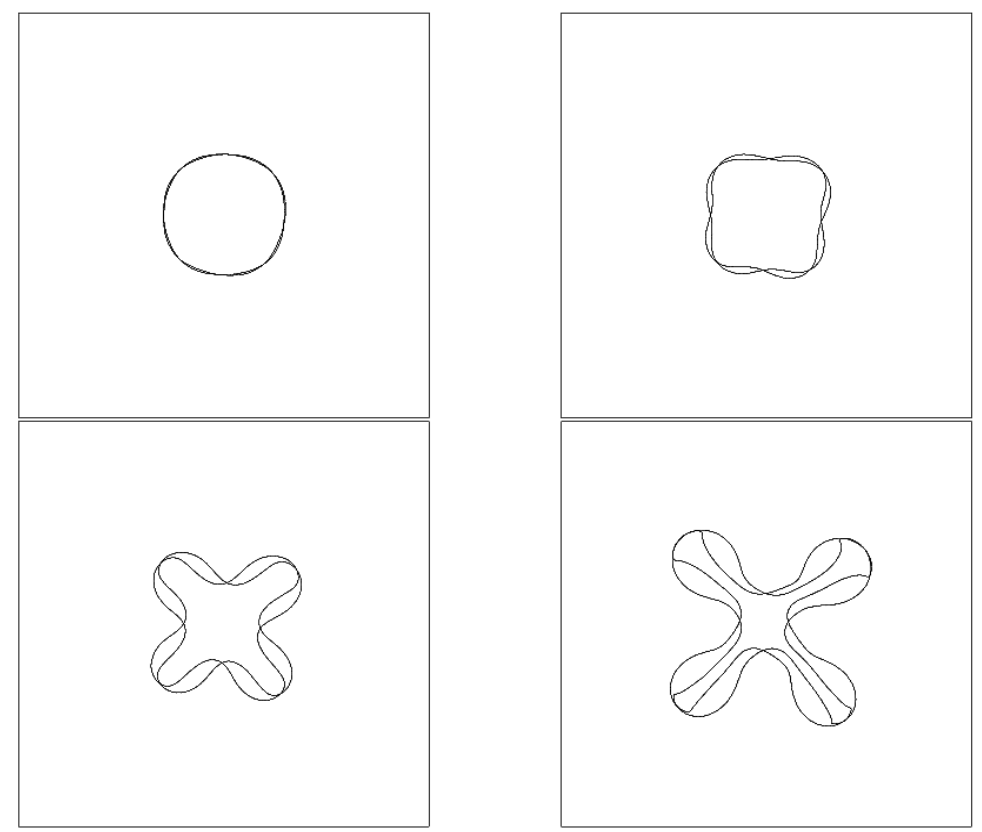

Figure 19. Horizontal maps of potential vorticity in layers 1 and 3 superimposed (contour $q_{j}= \pm 2 \pi$ ) for $r / h_{v}=11.9, d / h_{=} 4 / 3$, with initial superposition of equal amplitude mode 2 to 4 perturbations. Times shown range from $t=24$ to $t=48$ with increment $\delta t=8$, from left to right, and from top to bottom. 


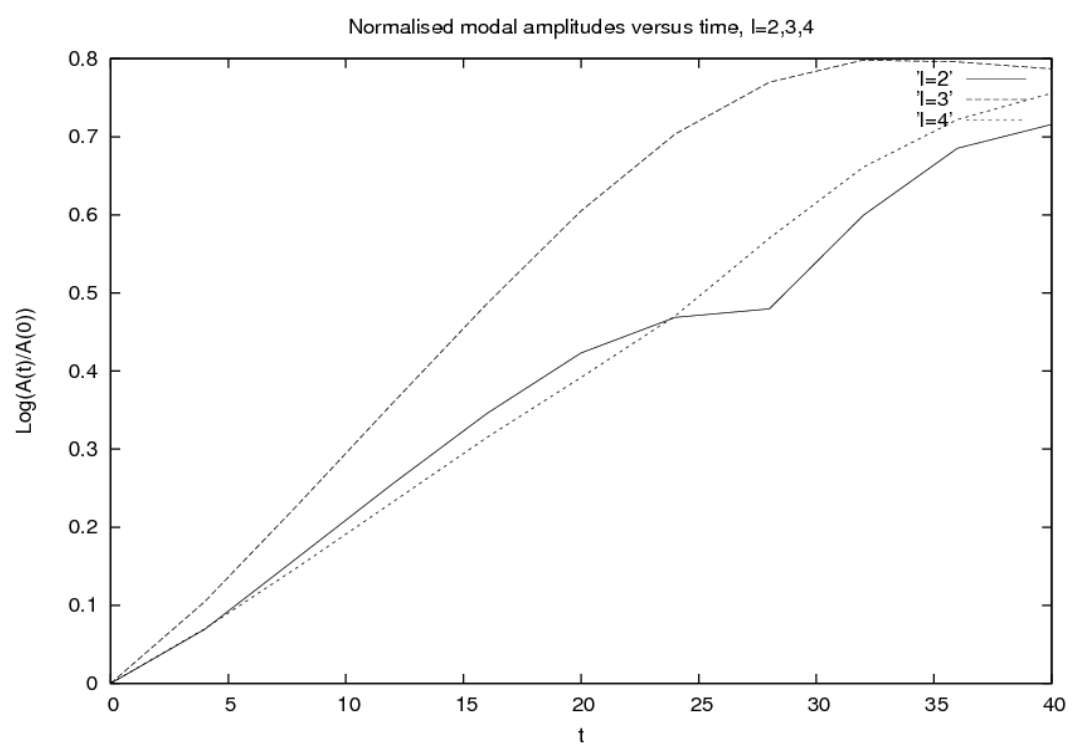

Figure 20. Time evolution of the amplitudes of the $l=2-4$ components of the perturbation in the instability of the $r / h_{v}=8.5, d / h_{v}=4 / 3$ vortex.

vortex amplitude, the long-range influence is mostly due to this latter, at least for several linear growth periods. But the mean flow has no barotropic component here, so that its decay scale is given by the largest deformation radius (which is unity). Therefore, for several linear periods, the influence of the image vortex due to domain periodicity, is negligible.

Thus, the most likely explanation for the asymmetric evolutions of the baroclinic vortex in these regions of the parameter plane, is the similarity of the growth rates. Indeed, another series of nonlinear simulations, performed with only the most unstable wave in the initialisation, leads to breaking into more symmetric hetons.

In all cases, as $d / h_{v}$ becomes larger, PV-containing layers become more distant and couple less strongly; also the volume integral of PV in the uppermost and lowermost layers decreases with their thicknesses. Then, baroclinic instability becomes weaker and unstable wavenumbers decrease for given $r / h_{v}$.

These results can be compared with those of figure 9 . For $d / h_{v}=2.0$, the appearance of mode 2 is shifted to $r / h_{v}=4$ and the predominance of mode 3 to $r / h_{v}=9$. Nevertheless, mode 4 still exists for $r / h_{v}=10$, whereas it was not observed in the linear analysis of continuously stratified hetons.

The time evolution of the perturbed hetons is shown on figures 17-19. The initial perturbation for each simulation is a superposition of equal-amplitude modes $l=2, l=3$ and $l=4$ which are the dominant modes in the parameter region considered here. The scaled vortex radii are $r / h_{v}=5.1,8.5,11.9$, and $d / h_{v}=4 / 3$ for the three simulations.

In the first example (figure 17), mode $l=2$ is most unstable and the circular vortex destabilises to form two hetons propagating in opposite directions as in figure 4 . The vertical phase difference, opposite to the vertical shear of mean azimuthal velocity, can be seen, characteristic of baroclinic instability. In the second example (figure 18), mode $l=3$ is most unstable, and modes $l=2$ and $l=4$ are slightly less unstable. This leads 
to an asymmetric breaking into three hetons. Figure 20 illustrates the time evolution of the $l=2$ to $l=4$ components of the perturbation, obtained by Fourier analysis, in this example. Clearly, mode $l=3$ is most unstable and is followed by modes $l=2$ and $l=4$ which have similar growth rates. Comparison of early growth rates in the nonlinear model (during the linear stage) with linear growth rates is satisfactory. The final growth of perturbation components with high wavenumber in the final stage is also related to the pinching of vorticity contours (formation of filaments) before final breaking. In the third example (figure 19), the evolution is very similar to that shown on figure 8 . The circular heton breaks into four hetons which propagate outwards. A remnant of the core, with square shape, is finally left at the centre (not shown). Other experiments, performed with increasingly large vortices indicate that this central remnant can later become unstable and break into dipoles (a result previously obtained by M. Sokolovskiy, priv. comm.).

\subsection{Influence of the vertical structure of the flow}

Here we discuss our previous results in view of the structure and observed evolution of oceanic vortices.

In the previous simulations, no vortex, initially perturbed either with white noise vorticity, or with a superposition of $l=2,3,4$ disturbances, showed finite-amplitude stabilisation. On the contrary, when the upper and middle layers have equal thicknesses and when the lower layer is thicker, finite-amplitude stabilisation of the perturbation was often observed.

Results not shown here indicate that when the lower layer is twice as thick as the upper and middle layers, the outcome of simulations with mode $l=2$ perturbation is often a baroclinic tripole (an elliptical core vortex, surrounded by two crescent-shaped satellites, at right angle to the core vortex). This result has already been obtained in two-layer models (Sokolovskiy and Verron (2000)). The time evolution of such vortices shows a marked difference between the upper and lower layers, when potential vorticity resides. The upper layer vortex breaks rapidly, but the lower layer vortex is less deformed. Indeed, the volume integral of potential vorticity for the lower layer is twice as large as for the upper layer. This induces stronger shear velocities in the upper layer, but weaker ones in the lower layer. The final stabilisation of the perturbation at finite amplitude is due to the absence of dipolar breaking of the lower layer vortex. In fact, the vorticity anomalies in the final state, are then confined within a separatrix, as previous work has shown (Carton and Corréard (1999)).

To create radially propagating hetons, the mutual influence of the dynamically active layers (where potential vorticity is located), must be comparable. This conclusion is supported by another set of experiments, where the potential vorticity amplitude is doubled in the upper layer. Then, the volume integral of potential vorticity is equal for the upper and lower layers, and hetonic breaking is recovered in all cases.

Thin upper layers and strong motion in these layers are representative of oceanic motions. The vortices studied above have radii ranging mostly between 3 and 5 deformation radii, or, at mid-latitudes, between 90 and $150 \mathrm{~km}$ (we consider here the first internal radius of deformation). This size is characteristic of intense rings of western boundary currents. Such rings have not yet been reported to split into hetons. The influence of a barotropic component, or the nonlinearity of the rings (i.e. their lens-like structure), may account for this difference in behaviour. In a two-layer shallow-water model, it was shown that the addition of a barotropic component to the mean flow can reduce the instability or even stabilise an otherwise unstable vortex (Dewar and Gailliard (1994)). In the same model, baroclinic anticyclones with strong isopycnal deviations (i.e. lens eddies) were 
shown to be linearly more stable than their quasi-geostrophic counterparts (Baey and Carton (2002)).

\section{Conclusions}

In this paper, the linear stability of quasi-geostrophic hetons was studied and their nonlinear evolution was investigated, in two distinct frameworks. On the one hand, using the equations governing a continuously stratified fluid, we discretised the domain in the vertical with many layers (typically about $\mathcal{O}(100)$ layers spanning the heton). This allows many baroclinic modes to develop.

On the other hand, we have contrasted these results with results obtained with a 3-layer model. Here the barotropic and the two first baroclinic modes are taken into account. Most of previous studies restrained attention to 2-layers models where the barotropic mode and the first baroclinic mode only can exist.

We have first shown a remarkable similarity in linear instability properties of hetons in the continuously stratified and in 3-layer models, when the vertical gap between the two vortices is finite. The instability threshold for the various modes, in terms of vortex radii, and the growth rates for the normal mode perturbations, are quite comparable. The nonlinear evolutions of perturbed and unstable hetons in the two models are also similar.

Secondly, we have shown that the vertical offset between the two poles of the heton has a significant influence on its stability, especially when $d / h_{v} \longrightarrow 0$. The smaller the gap $d$ is, the more the deformation of the heton's poles depends on height. This inhomogeneity cannot be captured by a few-layer model. It must be noted that the 3-layer model cannot accommodate both constant $N$ and vertically symmetric layer thicknesses for $H_{2}<0.25$ (or $d<\pi / 4$ ) and thus cannot, under such conditions, simulate infinitesimal gaps. In the continuous stratification case, when $d / h_{v}$ is increased, the vertical scale of the deformation increases (it is less concentrated vertically). Then the layered model and continuous stratification model results converge. The 3-layer model, which is less computer-time consuming can accurately describe the characteristics of the flow in these cases.

In the three-layer model, nonlinear simulations with vortices perturbed either with white noise vorticity, or with a superposition of modal disturbances, evolve according to the linear prediction (i.e. they break on the linearly most unstable mode), if one mode is noticeably more unstable than the others; when two modes have comparable growth rates, the dominant mode at the end of the simulation is unpredictable. The three-layer model provide vortex evolutions similar to those observed in a continuously stratified fluid, in the same region of parameter space. The three-layer model indicates that the presence of a barotropic component in mean potential vorticity, which asymmetrises the mutual influences between layerwise vortices, leads to a nonlinear stabilisation of linearly unstable vortices.

We have also discovered a very small region of the parameter space where a baroclinic $l=1$ mode is unstable. This instability domain is confined in a region $d / h, r / h \ll 1$. Therefore, these modes are not captured by the 3-layer model (see above). However, the nonlinear evolution in the continuously-stratified case shows that these modes are weak in the sense that they do not destroy the vortices. The instability generates a very small amount of filaments, and mostly misaligns the vortices. The consequence of this misaligment is a slow translation of the heton.

Overall, many hetons were found strongly unstable in the continuously stratified QG model, despite being separated in the vertical. Only well separated hetons $(d / h \sim \mathcal{O}(1)$, 
or greater with $r / h \sim \mathcal{O}(1)$ or smaller) are stable. Other (isolated) hetons break into smaller vortices, the number of which depends on the azimuthal wavenumber of the most unstable mode.

This observation has to be extended to more general models, in particular models coupling interior and surface dynamics (like the coupled QG-Surface QG model), or models allowing higher Rossby numbers and inertia-gravity waves (like the primitive equation and the Boussinesq models) to draw firmer conclusions for oceanic and atmospheric flows. In particular, the analysis of oceanic vortices indicates that their stability is strongly dependent on their nonlinearity. This conclusion was reached in a two-layer shallow-water model. A generalisation of the present study to three-layer shallow-water models and to continuously stratified primitive-equations model should be carried out in that respect.

\section{Appendix A. Stratification and vertical modes in the layered model}

The three-layer model used here has rigid top and flat bottom boundary conditions (with free-slip conditions and zero density anomaly). This will not induce major differences with the continuously stratified model where vertical periodicity effects were avoided.

In a continuously stratified quasi-geostrophic model with rigid top and bottom, and constant $f_{0}$ and $N_{0}$, the eigenvalues of the stratification operator $\left(f_{0} / N_{0}\right)^{2} \partial_{z}^{2}$ are $n \pi / H$ and the associated eigenvectors are

$$
\psi=\psi_{n} \cos (n \pi z / H),
$$

where $n=0$ denotes the barotropic mode. Hereafter, the vertical scale is normalised by $\pi$ to have unit total depth; therefore, stratification eigenvalues are equal to $n$.

In the three-layer model, the barotropic and first two baroclinic modes are represented. To compute potential vorticity, we have to determine the $F_{j}$ with the knowledge of the stratification eigenvalues $\gamma_{1}=1$ and $\gamma_{2}=2$, and of the layer thicknesses. Note that, with the rigid lid, $\gamma_{0}=0$ for the barotropic mode. The two deformation radii are thus $R_{d 1}=1, R_{d 2}=1 / 2$. Diagonalising the $F$ matrix provides $g_{1}^{\prime}$ and $g_{2}^{\prime}$ via

$$
Y_{3}=Y_{1} / g_{1}^{\prime}+Y_{2} / g_{2}^{\prime}, \quad Y_{4}=1 /\left(g_{1}^{\prime} g_{2}^{\prime}\right),
$$

with $Y_{1}=H_{3}\left(1-H_{3}\right), Y_{2}=H_{1}\left(1-H_{1}\right), Y_{3}=H_{1} H_{2} H_{3}\left(F_{1}+F_{2}+F_{3}+F_{4}\right), Y_{4}=$ $H_{1} H_{2} H_{3}\left(F_{1} F_{3}+F_{1} F_{4}+F_{2} F_{4}\right)$, again using $f_{0}=1, H=1$. The eigenmodes (vertical modes) are solutions of $G X=0$ with

$$
G=\left(\begin{array}{ccc}
-\gamma_{1,2}^{2}+F_{1} & -F_{2} & 0 \\
-F_{1} & -\gamma_{1,2}^{2}+F_{2}+F_{3} & -F_{4} \\
0 & -F_{3} & -\gamma_{1,2}^{2}+F_{4}
\end{array}\right) .
$$

Numerical solutions for various values of $H_{j}$ show that the barotropic mode is likesigned on all layers, the first baroclinic mode changes sign once and the second baroclinic mode twice vertically, as expected. We call $A=\left(a_{i j}\right)$ the layer to mode transition matrix, that is $\psi_{m}=a_{m i} \psi_{i}$, and $B=\left(b_{i j}\right)$ its inverse.

In vertical modes, potential vorticity is written

$$
\begin{gathered}
q_{t}=\nabla^{2} \psi_{t}=\sum_{j} a_{1 j} q_{j}, \\
q_{c 1}=\nabla^{2} \psi_{c 1}-\gamma_{1}^{2} \psi_{c 1}=\sum_{j} a_{2 j} q_{j},
\end{gathered}
$$




$$
q_{c 2}=\nabla^{2} \psi_{c 2}-\gamma_{2}^{2} \psi_{c 2}=\sum_{j} a_{3 j} q_{j}
$$

which allows its inversion into streamfunction.

It must be remarked that imposing vertical symmetry in layer thicknesses and constant $N$ does not allow three-layer analogues of the continuously stratified models, for $H_{2}<0.25$. Therefore, the case of vertically close vortices in the heton is not addressed in the threelayer model.

\section{Appendix B. Linearised potential vorticity equations in the layered model}

The linearised potential vorticity $(\mathrm{PV})$ equation is

$$
\partial_{t} q_{j}^{\prime}+\frac{U_{j}}{r} \partial_{\theta} q_{j}^{\prime}+\frac{1}{r} \frac{d Q_{j}}{d r} \partial_{\theta} \psi_{j}^{\prime}=0
$$

Here $Q_{j}$ is not continuous and this equation has to be integrated in the vicinity of each PV front (at radius $r$ ). This integrated equation is equivalent to the continuity of the total tangential velocity at the PV front inserted into the kinematic equation of the PV front. We follow this alternative procedure here.

The azimuthal velocity of the circular vortex at the vortex radius $r$ is

$$
U_{t}(r)=q_{t} r / 2, U_{c 1}(1)=q_{c 1} I_{1}\left(\gamma_{1} r\right) K_{1}\left(\gamma_{1} r\right), U_{c 2}(1)=q_{c 2} I_{1}\left(\gamma_{2} r\right) K_{1}\left(\gamma_{2} r\right) .
$$

The perturbation streamfunction is a normal mode

$$
\psi_{j}^{\prime}=\phi_{j}(\rho) \exp (i l(\theta-c t)),
$$

where $\rho$ is the generic radial coordinate. Here $l$ the azimuthal mode, $c_{r}=R e(c)$ the phase speed of the perturbation and $\sigma_{r}=l c_{i}=l \operatorname{Im}(c)$ its growth rate.

The perturbation potential vorticity is a Dirac at the PV front $(\rho=r)$.

Using continuity of the perturbation streamfunction at the front, the internal/external solutions in modal perturbation streamfunction, are

$$
\begin{gathered}
\phi_{t}(\rho)=A_{1}(\rho / r)^{l}, A_{1}(\rho / r)^{-l}=A_{1} \phi_{1 i}(\rho), A_{1} \phi_{1 e}(\rho), \\
\phi_{c 1}(\rho)=A_{2} I_{l}\left(\gamma_{1} \rho\right) K_{l}\left(\gamma_{1} r\right), A_{2} I_{l}\left(\gamma_{1} r\right) K_{l}\left(\gamma_{1} \rho\right)=A_{2} \phi_{2 i}(\rho), A_{2} \phi_{2 e}(\rho), \\
\phi_{c 2}(\rho)=A_{3} I_{l}\left(\gamma_{2} \rho\right) K_{l}\left(\gamma_{2} r\right), A_{3} I_{l}\left(\gamma_{2} r\right) K_{l}\left(\gamma_{2} \rho\right)=A_{3} \phi_{3 i}(\rho), A_{3} \phi_{3 e}(\rho) .
\end{gathered}
$$

At $\rho=r$, we write their values symbolically as $A_{m} \phi_{m}(r), m=1,2,3$.

The continuity of total azimuthal velocity $u_{\theta}^{\prime}+\eta\left(d U_{\theta} / d r\right)$ at $\rho=r$, where $\eta$ is the contour perturbation, leads to

$$
\sum_{m} b_{j m} A_{m}\left(\partial_{r} \phi_{m e}-\partial_{r} \phi_{m i}\right)=\eta_{j} q_{j}
$$

In more compact form, this is written

$$
\eta_{j}=\sum_{m} M_{j m} A_{m}
$$

with $M_{j m}=b_{j m}\left(\partial_{r} \phi_{m e}-\partial_{r} \phi_{m i}\right)$, and the reciprocal equation is

$$
A_{m}=\sum_{j} N_{m j} \eta_{j}
$$


with $N=M^{-1}$. With normal modes, the kinematic equation at the front is, in each layer

$$
\left(U_{j}(r)-c\right) \eta_{j}=\phi_{j}(r)
$$

Now

$$
\phi_{j}(r)=\sum_{m} b_{j m} A_{m} \phi_{m}(r)=\sum_{m k} b_{j m} \phi_{m}(r) N_{m l} \eta_{k}
$$

and thus

$$
U_{j}(r) \eta_{j}-\sum_{m k} b_{j m} \phi_{m}(r) N_{m k} \eta_{l}=c \eta_{j}
$$

Finally, linear stability is given by the system

$$
L_{j k} \eta_{k}=c \eta_{j}
$$

with

$$
L_{j k}=U_{j} \delta_{j k}-\sum_{m} b_{j m} \phi_{m}(r) N_{m k}
$$

\section{REFERENCES}

BAey, J. \& CARton, X. 2002 Vortex multipoles in two-layer rotating shallow-water flows. J. Fluid Mech. 460, 151-175.

Bambrey, R. R., Reinaud, J. N. \& Dritschel, D. G. 2007 Strong interactions between two co-rotating quasi-geostrophic vortices. J. Fluid Mech. 592, 117-133.

Bane, J., O'Keefe, L. \& WATts, D. 1999 Mesoscale eddies and submesoscale coherent vortices: Their existence near and interactions with the gulf-stream. Mesoscale/Synoptic Coherent Structures in Geophysical Turbulence; Proceedings of the 20th International Liege Colloquium on Ocean Hydrodynamics. Elsevier, New York, 501-518.

Carton, X., Chérubin, L., Paillet, J., Morel, Y., Serpette, A. \& Le Cann, B. 2002 Meddy coupling with a deep cyclone in the gulf of cadiz. J. Mar. Syst. 32, 13-42.

Carton, X. \& Corréard, S. 1999 Baaroclinic tripolar geostrophic vortices. Proceedings of the IUTAM Symposium "Simulation and identification of organized structures in flows" Kluwer Acad. Publ., 181-190.

CorréARD, S. \& CARTON, X. 1999 Vertical alignment of geostrophic vortices: On the influence of the initial distribution of potential vorticity. Proceedings of the IUTAM Symposium "Simulation and identification of organized structures in flows" Kluwer Acad. Publ., 191-200.

Deem, G. \& Zabusky, N. 1978 Vortex waves: Stationary "v states', interactions, recurrence and breaking. Phys. Rev. lett. 40, 859-862.

Dewar, W. \& Gailliard, C. 1994 The dynamics of barotropically dominated rings. J. Phys. Ocenogr. 24, 5-29.

DritsChel, D. 1986 The nonlinear evolution or rotating configurations of uniform vorticity. $J$. Fluid Mech. 172, 157-182.

Dritschel, D. \& WAUGh, D. 1992 Quantification of the inelastic interaction of two asymmetric vortices in two-dimensional vortex dynamics. Phys. Fluids A 4, 1737-1744.

Dritschel, D. G. 2002 Vortex merger in rotating stratified flows. J. Fluid Mech. 455, 83-101.

Dritschel, D. G. \& Ambaum, M. H. P. 1997 A contour-advective semi-lagrangian algorithm for the simulation of fine-scale conservative fields. Q. J. R. Met. Soc. 123, 1097-1130.

Dritschel, D. G. \& DE LA Torre JuÁrez, M. 1996 The instability and breakdown of tall columnar vortices in a quasi-geostrophic fluid. J. Fluid Mech. 328, 129-160.

FlierL, G. 1988 On the instability of geostrophic vortices. J. Fluid Mech. 197, 339-388.

Griffiths, R. \& Hopfinger, E. 1987 Coalescing of geostrophic vortices. J. Fluid Mech. 178, 73-97.

von Hardenberg, J., McWilliams, J. C., Provenzale, A., Shchpetkin, A. \& Weiss, J. B. 2000 Vortex merging in quasi-geostrohic flows. J. Fluid Mech. 412, 331-353. 
Helfrich, K. \& Send, U. 1988 Finite-amplitude evolution of two-layer geostrophic vortices. J. Fluid Mech. 197, 331-348.

Hogg, N. G. \& Stommel, H. M. 1985 The heton, an elementary interaction between discrete baroclinic geostrophic vortices, and its implications concerning eddy heat flow. Proc. $R$. Soc. Lond. A 397, 1-20.

Melander, M., Zabusky, N. \& MCWilliams, J. 1987 Asymmetric vortex merger in two dimension: Which vortex is "victorious" ? Phys. Fluids A 30, 2610-2612.

Melander, M., Zabusky, N. \& McWilliams, J. 1988 Symmetric vortex merger in two dimensions: causes and conditions. J. Fluid Mech. 195, 303-340.

Miyazaki, T., Yamamoto, M. \& Fujishima, S. 2003 Counter-rotating quasigeostrophic ellipsoidal vortex pair. J. Phys. Soc Jpn. 72 (8), 1942-1953.

Overman, E. \& Zabusky, N. 1982 Evolution and merger of isolated vortex structures. Phys. Fluids 25, 1297-1305.

Ozugurlu, E., Reinaud, J. N. \& Dritschel, D. G. 2008 Interaction between two quasigeostrophic vortices of unequal potential-vorticity. J. Fluid Mech. 597, 395-414.

Polvani, L. 1991 Two-layer geostrophic vortex dynamics. part 2. alignment and two-layer vstates. J. Fluid Mech. 225, 241-270.

Polvani, L., Zabusky, N. \& Flierl, G. 1989 Two-layer geostrophic vortex dynamics. part 1. upper-layer v-states and merger. J. Fluid Mech. 205, 215-242.

Reinaud, J. N. \& Dritschel, D. G. 2002 The merger of vertically offset quasi-geostrophic vortices. J. Fluid Mech. 469, 287-315.

Reinaud, J. N. \& Dritschel, D. G. 2008 Destructive interactions between two counterrotating quasi-geostrophic vortices. J. Fluid Mech. submitted.

Robinson, A. 1983 Eddies in marine science. Springer Verlag.

Serra, N., Ambar, I. \& Käse, R. 2005 Observations and numerical modelling of the mediterranean outflow splitting and eddy generation. Deep-Sea Res. 52, 383-408.

Sokolovskiy, M. 1997 Stability of an axisymmetric three-layer vortex. Izvestiya, Atmospheric and Oceanic Physics 33, 16-26.

Sokolovskiy, M. \& Verron, J. 2000 Finite-core hetons: stability and interactions. J. Fluid Mech. 423, 127-154.

VAlcke, S. \& Verron, J. 1993 On interactions between two finite-core hetons. Phys. Fluids, A 5, 2058-2060.

VAluis, C. 2006 Atmospheric and Oceanic Fluid Dynamics: Fundamentals and Large-scale Circulation. Cambridge University Press.

Verron, J., Hopfinger, E. \& McWilliams, J. 1990 Sensitivity to initial conditions in the merging of two-layer baroclinic vortices. Phys. Fluids A 2, 886-889.

Verron, J. \& VAlcke, S. 1994 Scale-dependent merging of baroclinic vortices. J. Fluid Mech. 264, 81-106.

VIERA, F. 1995 On the alignment and axisymmetrization of a vertically-tilted geostrophic vortex. J. Fluid Mech. 289, 29-50.

YAsudA, I. \& FlieRL, G. 1995 Two-dimensional asymmetric vortex merger: Contour dynamics experiments. J. Oceanogr. 51, $145=-170$.

YASUdA, I. \& FlieRL, G. 1997 Two-dimensional asymmetric vortex merger: merger dynamics and critical merger distance. Dyn. Atmos. Oceans 26, 159-181. 